\title{
Effects Comparison of Different Resilience Enhancing Strategies for Municipal Water Distribution Network: A Multidimensional Approach
}

\author{
Xudong Zhao, Zhilong Chen, and Huadong Gong \\ State Key Laboratory of Explosion \& Impact and Disaster Prevention \& Mitigation, \\ PLA University of Science and Technology, Nanjing 210007, China \\ Correspondence should be addressed to Huadong Gong; gonghd2006@163.com
}

Received 5 May 2015; Revised 7 July 2015; Accepted 9 July 2015

Academic Editor: Anna Vila

Copyright $\odot 2015$ Xudong Zhao et al. This is an open access article distributed under the Creative Commons Attribution License, which permits unrestricted use, distribution, and reproduction in any medium, provided the original work is properly cited.

\begin{abstract}
Water distribution network (WDN) is critical to the city service, economic rehabilitation, public health, and safety. Reconstructing the WDN to improve its resilience in seismic disaster is an important and ongoing issue. Although a considerable body of research has examined the effects of different reconstruction strategies on seismic resistance, it is still hard for decision-makers to choose optimal resilience enhancing strategy. Taking the pipeline ductile retrofitting and network meshed expansion as demonstration, we proposed a feasible framework to contrast the resilience enhancing effects of two reconstruction strategies-units retrofitting strategy and network optimization strategy-in technical and organizational dimension. We also developed a new performance response function (PRF) which is based on network equilibrium theory to conduct the effects comparison in integrated technical and organizational dimension. Through the case study of municipal WDN in Lianyungang, China, the comparison results were thoroughly shown and the holistic decision-making support was provided.
\end{abstract}

\section{Introduction}

As an important part of urban lifelines [1], the municipal water distribution network (WDN) is playing a significant role in keeping economic growth and improving citizens' live quality in modern society [2]. For a long time, the safety of municipal WDNs in earthquakes has been one of the important issues in the field of civil engineering. Since the 20th century, there have been about 1200 earthquakes with at least magnitude 7.0 or greater, which have caused severe damage to municipal water distribution networks. Some recent seismic disasters-8.0-magnitude quake in Wenchuan, China (2008) [3], and 9.0-magnitude quake in East Japan (2011) [4] - have also raised great concern about the safety of municipal water distribution networks in earthquake.

For safeguarding the municipal water distribution network in seismic disaster, there are several reconstruction strategies that can be conducted by the city administrators. These reconstruction measures can be divided into two categories: units retrofitting strategy and network optimization strategy. Units retrofitting strategy focuses on the measures that will improve the antiseismic capability of unit components in WDN, such as ductile retrofitting of pipelines [5], structure reinforcement of water treatment plant [6], and power backup of pumping station. Among these, ductile retrofitting of pipelines is a typical measure that can be widely used in WDNs [5], while, for the network optimization strategy, the concern is on improving the network performance to withstand the seismic disaster, such as the meshed expansion of network [7], design for redundancy [8], and self-healing and self-adapting system response mechanisms [9]. Among these measures, meshed expansion of network is frequently used and easy to implement [7].

Actually, the effect of ductile retrofitting strategy of pipelines on seismic resistance has been validated by the real events such as the 6.3-magnitude quake that hit L'Aquila, Italy, in 2009 [5]. Yazdani et al. have also studied the effect of network expansion measure for WDN in Kumasi, the second largest city in Ghana [7]. However, facing the constraints of local reconstruction budgets and resource, it is hard for 
decision-makers to make optimal decision because little research has addressed the effects comparison of these two strategies.

To conduct this comparison, concepts such as "risk" [1012], "reliability" [13-15], and "vulnerability" [16-18] can be used to assess the antiseismic capability of WDN under different reconstruction strategies. In recent years, the concept of "resilience" has also attracted increasing attention because of its application in evaluating the capability of urban lifelines to withstand various natural disasters [19-23]. The resilience originally refers to the ability of a system to absorb external disturbance and maintain expected performance. Bruneau et al. (2003) [19] and Chang and Shinozuka (2004) [23] established that, in a disaster, urban infrastructure resilience is a measurable concept with four interrelated dimensions (technical, organizational, social, and economic). Further, resilience can be comprehensively assessed if three key features are considered, namely, the probability of a disaster's occurrence, the consequences of that disaster, and the recovery time of the infrastructure system.

Therefore, for municipal water distribution networks, effects comparison of different reconstruction strategies on improving the network resilience should be conducted in multidimensions, namely, the technical, organizational, social, and economic dimension.

Technical resilience refers to how well physical systems perform when subjected to earthquake forces [19]. Chang and Shinozuka (2004) [23] use the number of major pumping stations lost and the percentage of pipes broken to measure technical performance of WDN [23]. Organizational resilience refers to the ability of organizations to respond to emergencies and carry out critical functions [19]. Chang and Shinozuka (2004) [23] use the percentage of population losing water service to measure the organizational performance of WDN. Social resilience refers to the capacity to reduce the negative societal consequences of loss of critical services in earthquakes. Economic resilience refers to the ability to reduce the direct and indirect economic losses of community resulting from earthquakes [19]. In the study of Chang and Shinozuka (2004) [23], social performance was measured by the population of community displaced from their homes due to water outage, while economic performance was measured by the loss of gross regional product (GRP).

According to the above definition of four resilience dimensions, Chang and Shinozuka (2004) have concluded that the technical and organizational dimensions are most pertinent to the performance and resilience of infrastructure systems such as WDN and electric power grid, while the social and economic dimensions are most relevant to the performance and resilience of the community as a whole [23]. The aim of this study is to choose optimal resilience enhancing strategy for WDN itself; it is reasonable to limit our research to the technical and organizational dimensions which are most pertinent to the performance and resilience of infrastructure system.

In general, previous studies have only used one dimension to assess resilience. Yazdani et al. (2011) have contrasted the effects of different reconstruction measures on improving the WDN resilience [7]. In their study, the effect comparison mostly focused on the physical network metrics and measurements, namely, the technical dimension. Ouyang and Dueñas-Osorio (2014) assessed the hurricane resilience of electric power systems in each dimension, respectively [21]. However, as we outlined earlier, the dimensions of resilience are interrelated. It is difficult to fully reflect resilience using single dimension, respectively; effects comparison results obtained from different dimensions may be inconsistent or even contradictory. Therefore, besides each dimension, respectively, it is also necessary to develop a comprehensive metric or measurement that can integrate the characteristics of multiple dimensions and produce consistent and holistic results for decision-makers.

To realize a comprehensive effect comparison of different resilience improving strategies for WDNs, this study differs from previous research in the following two aspects:

(1) Taking the ductile retrofitting and the network meshed expansion as typical demonstration measures, we addressed the effect comparison of two reconstruction strategies-units retrofitting strategy and network optimization strategy-on improving the seismic resilience of WDN.

(2) Not just in single dimension of resilience, this work conducted the effect comparison in both technical and organizational dimension. What is more, taking the interrelationship between these two dimensions into consideration, we also developed a measurement integrating these two dimensions to assess the resilience enhancing effect of these two strategies.

\section{Methodology and Framework}

2.1. Resilience Metric of WDN. In order to conduct the effects comparison of different reconstruction strategies on seismic resilience, it is necessary to assess the resilience of WDN after reconstruction. Broadly, the resilience of urban infrastructures can be assessed using three methods: the probabilistic analysis method [23], the expert judgment method [24], and the PRF (performance response function) method $[19,21,22$, 25]. Currently, the PRF method which uses the plotted curve of the relationship between network performance and time to assess resilience is the most used method.

After a seismic disaster, the recovery process can be represented by a PRF curve (Figure 1). An advantage of PRF method is that it can simultaneously show multiple key features of resilience, for example, disaster consequence, recovery degree, and recovery time. Disaster consequence, which depends on the absorbing and adapting capability of network to external shocks, indicates the decrease of network performance from the pre-event normal level to the lowest point after earthquake (e.g., $P_{0}$ to $P_{1}$ in Figure 1). Recovery degree, which is mainly restrained by the recovery budget (refers to the funds, repair materials, and other consumables), represents the final performance degree that system can achieve after restoration procedures [26]. Recovery time means the full time that the network needs to recover from disaster consequence (e.g., $t_{0}$ to $t_{R}$ in Figure 1). This key feature is determined by the recovery resource and the selected 


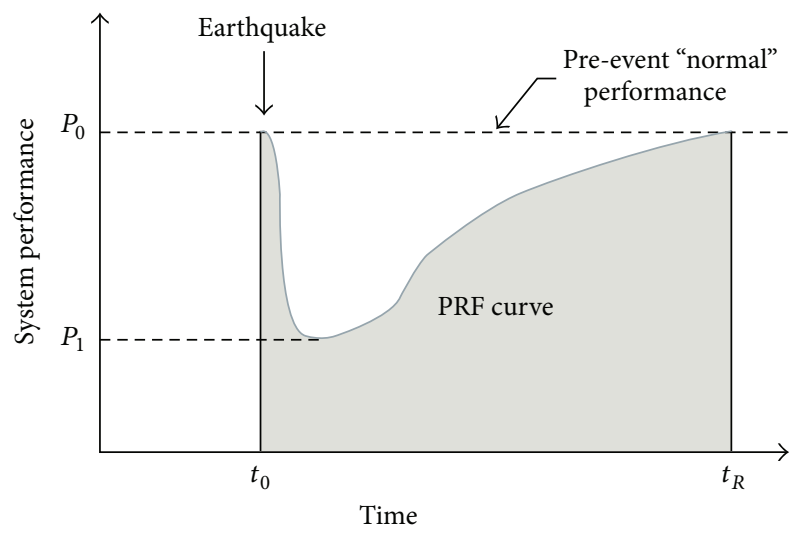

FIGURE 1: Typical illustration of performance response function (PRF) curve.

recovery strategy. Other than the recovery budget, recovery resource refers to the nonexpendable items such as repair equipment and human resource. Recovery strategy, which includes stochastic strategy and optimal strategy, refers to the resource allocation policy and repairing schedule.

According to the shape of PRF curve and the area under the curve, the resilience $R$ of an urban lifeline network can be formulated as $[19,20,22]$

$$
R=\frac{A_{\varepsilon}}{A_{p}}=\frac{\int_{t_{0}}^{t_{R}} \varepsilon(t) d t}{\int_{t_{0}}^{t_{R}} p(t) d t},
$$

where $\varepsilon(t)$ is the PRF of a lifeline network at seismic disaster. $p(t)$ is the normal performance level of the network (prior to any earthquake effects): this variable can be represented by a stochastic process or constant line [22], and in this study we used the latter (e.g., in Figure 1, it is the horizontal line through point $\left.P_{0}\right) . A_{\varepsilon}$ refers to the area under the $\varepsilon(t)$ curve from $t_{0}$ to $t_{R}$ (e.g., in Figure 1, this is the grey area under PRF curve) and $A_{p}$ is the area under the $p(t)$ curve from $t_{0}$ to $t_{R}$.

2.2. Overall Framework. On the basis of the PRF method, we developed a framework to contrast the seismic resilience of urban WDN under different reconstruction measures. In this framework, the resilience of three WDN states (original WDN, WDN under ductile retrofitting, and WDN under meshed expansion) was quantitatively compared. In addition, the contrast was conducted in each resilience dimension (technical dimension and organizational dimension), and a new PRF function based on network equilibrium theory was also proposed to conduct the comparison in integrated technical and organizational dimension. As shown in Figure 2, the resilience contrast procedure based on the overall framework can be divided into five steps.

Step 1. Determine the damage extent of each unit of three WDN states. This step can be accomplished by first analyzing the seismic intensity and ground motion parameters in a specific area and then substituting the results of the analysis into seismic fragility models of different unit kinds [27].
Step 2. For each WDN state, assess the damage extent of the whole system performance by using PRFs in different dimensions. Firstly, the PRFs were set in technical dimension and organizational dimension, respectively. Then a specific PRF based on network equilibrium theory was proposed to simultaneously integrate the characteristics of these two dimensions.

Step 3. Simulate the recovery process and plot the timedependent PRF curve of each WDN state with the constraints of the recovery conditions. In this step, the time-dependent PRF curve of each WDN state in different dimensions can be obtained.

Step 4. According to the time-dependent PRF curve of each WDN state, the resilience $R$ of each WDN state in different dimensions can be calculated.

Step 5. Contrast the resilience of each WDN state in different dimensions. Then evaluate the effects of two reconstruction strategies on improving the network resilience.

\section{Network PRF in Different Resilience Dimensions}

As we outlined above, the technical dimension and organizational dimension are research priorities in this study. In order to contrast the effects of reconstruction strategies for a WDN in different dimensions, it is significant to choose appropriate PRFs to represent each resilience dimension. Table 1 provides a brief illustration of the performance response functions (PRFs) utilized in this analysis together with an indication of which dimension is being assessed through each PRF.

3.1. Technical Dimension. In technical dimension, to represent physical status of the damaged system, the network performance is usually measured by the rate of physical unit loss [23] or the physical index of infrastructure networks $[7,22]$. Actually, a WDN is primarily a complex network of interconnected pipes and other units through which water is stored, conveyed, and pumped where necessary in order to meet the demand and pressure requirement of the system [7]. Throughout, this complex network can be represented as a mathematical graph $G=G(V, E)$ in which $V$ is the set of all graph nodes with $N$ elements (graph size) and $E$ is the set of graph edges with $m$ elements (graph order). In this work, the physical index of WDN introduced in technical dimension can be broadly classified from three perspectives: network structure, network redundancy, and network efficiency. The typical PRF from each perspective is provided below (corresponding to Table 1):

(1) $\varepsilon_{1}(t)$ refers to the link density variation during recovery process, which is the most fundamental indicator of the overall linkedness or sparseness of network structure [7]. $m(t)$ denotes the actual amount of graph edges at time $t$.

(2) $\varepsilon_{2}(t)$ refers to the meshedness coefficient variation during recovery process [28], which provides 


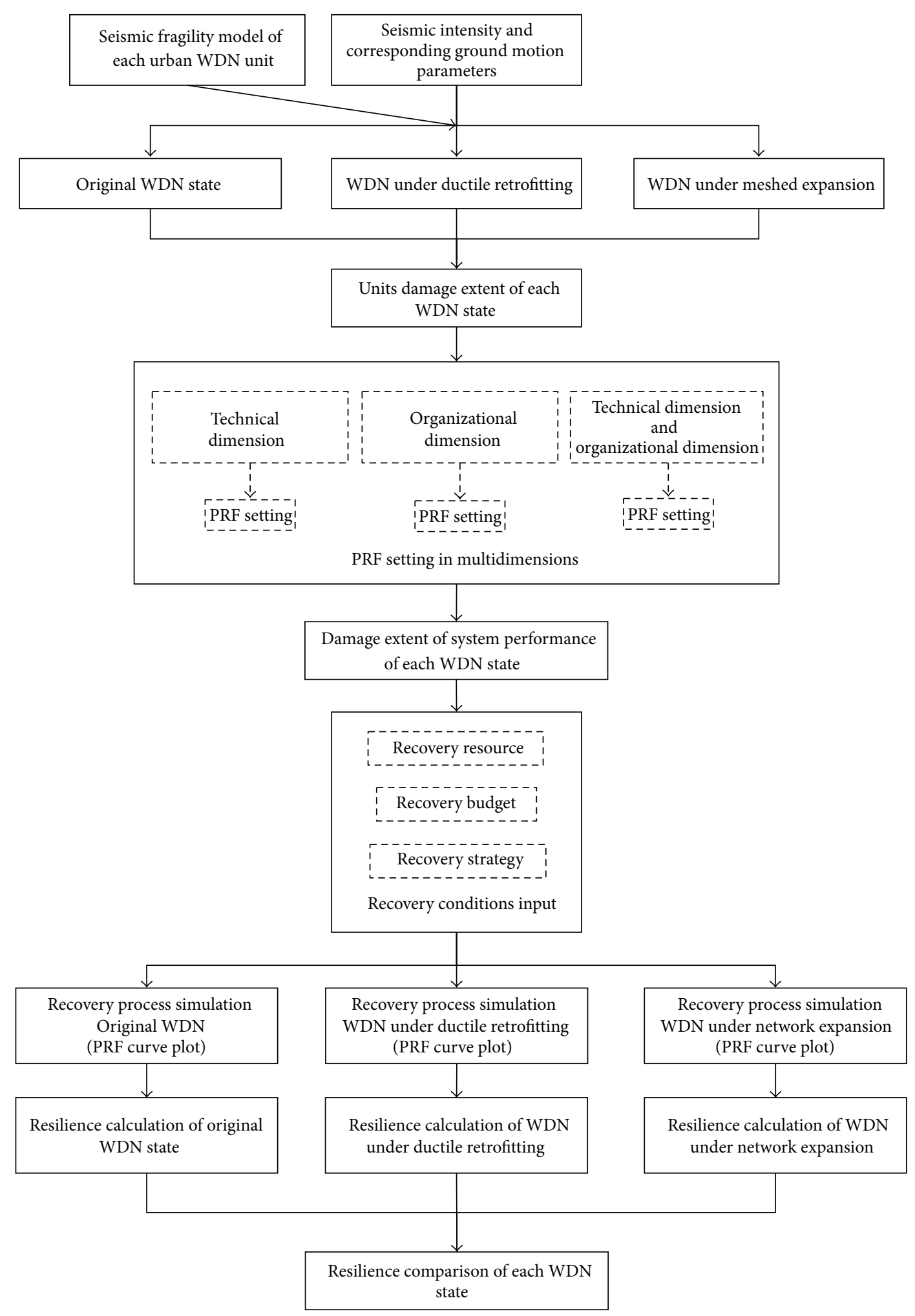

FIGURE 2: General framework to contrast the effects of different reconstruction strategies on seismic resilience. 
TABLE 1: PRF used to assess the resilience of WDNs in multidimensions.

\begin{tabular}{lll}
\hline Performance response function (PRF) & Measurement & Dimension \\
\hline$\varepsilon_{1}(t)=\frac{2 m(t)}{N(N-1)}$ & Link density [7] & Technical \\
$\varepsilon_{2}(t)=\frac{m(t)-N+1}{2 N-5}$ & Meshedness coefficient [28] & Average transmission-length [29] \\
$\varepsilon_{3}(t)=\frac{1}{n_{W}} \sum_{\omega \in W} \frac{1}{\lambda_{\omega}(t)}$ & Water supply rate & Organizational \\
\hline$\varepsilon_{4}(t)=\frac{1}{D_{W}} \sum_{\omega \in W} d_{\omega}(t)$ & $\begin{array}{l}\text { Network efficiency measure based on the } \\
\text { network equilibrium theory }\end{array}$ & Technical and organizational \\
\hline$\varepsilon_{5}(t)=\frac{1}{n_{W}} \sum_{\omega \in W} d_{\omega} / c_{\omega}(t)$ & &
\end{tabular}

an estimation of network redundancy in near-planar WDNs. $(m(t)-N+1)$ denotes the actually independent loops in the network at time $t$, while $(2 N-5)$ is the maximum possible loops in planar graph with $N$ nodes.

(3) $\varepsilon_{3}(t)$ refers to the average transmission-length variation during recovery process, which provides a limited view of network efficiency and reachability in water transmission [7, 29]. $\omega$ denotes $S / D$ (source/demand) node pair through which services are transmitted, $W$ is the set of all the $S / D$ pairs, and $n_{W}$ is the number of all the $S / D$ pairs. $\lambda_{\omega}(t)$ denotes the shortest path length between $S / D$ pair $\omega$ at time $t$.

3.2. Organizational Dimension. According to the study of Bruneau et al. [19], organizational resilience refers to the ability of organization to respond to emergencies and carry out critical function. One critical function of WDN which we focused on in the study is supplying the water service to residents. Therefore, in the study of Chang and Shinozuka (2004) [23], organizational performance of WDN refers to the extent of service disruption, measured as the percentage of population losing water service. Note that critical service function of infrastructure network is closely related to network flow conditions [23]. Cimellaro et al. also used network flow status to assess the resilience of natural gas distribution network in organizational dimension [25].

In our study, we used the extent of service disruption to reflect organizational performance as Chang and Shinozuka did [23]. But due to the lack of residential distribution data, we utilized the supply rate (the percentage of potable water amount that can be supplied) to measure the service function. The time-dependent PRF curve of water supply rate reflects the ability of WDN to respond to disaster and carry out critical service function under specific recovery conditions. The illustration of PRF is given below (corresponding to Table 1):

(4) $\varepsilon_{4}(t)$ refers to the water supply rate variation during recovery process. $D_{W}$ is the total potable water demand of the WDN. For any $S / D$ pair $\omega, d_{\omega}(t)$ denotes the potable water amount that can be satisfied at time $t$.
3.3. Technical Dimension and Organizational Dimension. For the resilience of infrastructure network, the technical and organizational dimensions are closely interrelated. In order to assess resilience in both dimensions, we defined a new PRF function as $\varepsilon_{5}(t)$ shows in Table 1:

(5) $\varepsilon_{5}(t)$ refers to the network efficiency variation during recovery process in both technical and organization dimension. In $\varepsilon_{5}(t), c_{\omega}(t)$ is named the "generic minimal path cost" [30] or "equilibrium travel disutility" [31] of $S / D$ pair $\omega$, which depends on the physical topology status of WDN at time $t ; d_{\omega}$ represents the demand for service that can be satisfied in $S / D$ pair $\omega$.

The PRF function defined in $\varepsilon_{5}(t)$ is derived from the N$\mathrm{Q}$ method presented by Nagurney and Qiang [30]. This method was developed on the basis of network equilibrium theory and applied to evaluate the performance of critical infrastructure networks.

According to network equilibrium theory, the generic minimal path cost $c_{\omega}(t)$ must follow Wardrop's first equilibrium principle [31], which can be described as follows:

$$
\begin{array}{ll}
c_{k}^{\omega}(t)=c_{\omega}(t) & \text { if } f_{k}^{\omega}(t)>0 \forall k \in K^{\omega}, \omega \in W \\
c_{k}^{\omega}(t) \geq c_{\omega}(t) & \text { if } f_{k}^{\omega}(t)=0 \forall k \in K^{\omega}, \omega \in W,
\end{array}
$$

where $k$ denotes a path that connects the source node and demand node in $S / D$ pair $\omega$, while $K^{\omega}$ is the set of all possible paths in $S / D$ pair $\omega ; f_{k}^{\omega}(t)$ is the service flow on path $k$ at time $t ; c_{k}^{\omega}(t)$ denotes the path cost on path $k$.

Figure 3 illustrates Wardrop's first equilibrium principle. For a network in the equilibrium condition, the path cost $c_{k}^{\omega}(t)$ for path $k$ equals the generic minimal path cost $c_{\omega}(t)$ for set $K^{\omega}$, provided that service flow $f_{k}^{\omega}(t)$ on path $k$ at time $t$ is greater than zero.

In $\varepsilon_{5}(t)$, the system performance in technical dimension is described by $c_{\omega}(t)$ to reflect the change of physical topology status during the recovery process. For the WDNs, $c_{\omega}(t)$ can be defined as the frictional head loss or the average transmission-length which are all fit to (2). The higher the efficiency of $\omega$ to transmit services, the lower the $c_{\omega}(t)$, while in organizational dimension, the system performance is described by $d_{\omega}$ that represents the demand for service that can be satisfied in $S / D$ pair $\omega$. When the connection 


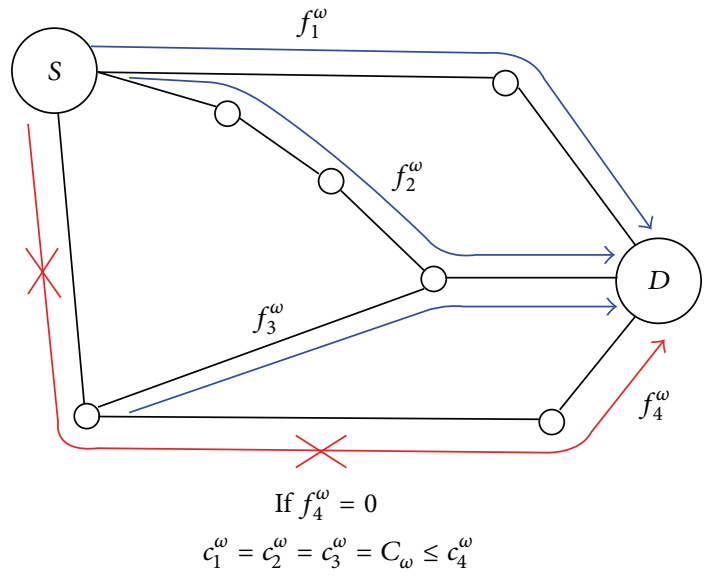

FIgURE 3: Wardrop's first equilibrium principle of network flow ( $S$ : source node, $D$ : demand node).

between source and demand nodes is broken, $c_{\omega}(t)$ tends to be infinity $(+\infty)$ and $d_{\omega} / c_{\omega}(t)$ approaches zero, which means the services cannot be transmitted to users.

\section{Case Study}

To illustrate our proposed approach, we used the municipal WDN in Lianyungang, Jiangsu province, China, as a case study. Under different reconstruction scenario of this WDN (e.g., original network, meshed expansion, and ductile retrofitting), we thoroughly investigated how resilience varied with the variation of recovery parameters (e.g., recovery budgets, recovery resources). In this way, we can compare the effects of two reconstruction strategies on resilience and propose several practical suggestions for supporting the decision-making processes that underpin network reconstruction efforts.

4.1. Case Background Information. Lianyungang, a city located in the northeast of Jiangsu province, on the Haizhou Gulf, is a key earthquake region of eastern China. As shown in Figure 4, the municipal water supply network in Lianyungang has two source nodes (water treatment plants $S_{1}$ and $S_{2}$ ) and 74 demand nodes distributed in the main urban area. According to the data provided by the Municipal Design and Research Institute of Lianyungang City, the total length of the arterial pipelines in the network is $171.2 \mathrm{~km}$, and the total demand of the network reaches $46.96 \times 10^{4} \mathrm{~m}^{3} /$ day.

4.2. Ductile Retrofitting and Meshed Expansion. As a widely used units retrofitting strategy, ductile retrofitting means to replace all pipelines which are made of brittle materials with those made of ductile materials. Pipelines of WDN are commonly made of concrete (prestressed or reinforced), asbestos cement, ductile iron, cast iron, steel, or plastic [27]. Among these materials, asbestos cement, concrete, and cast iron are known as brittle materials, while steel, ductile iron, and plastic (PVC) usually belong to ductile materials. In the present WDN of Lianyungang City, only 25 percent of

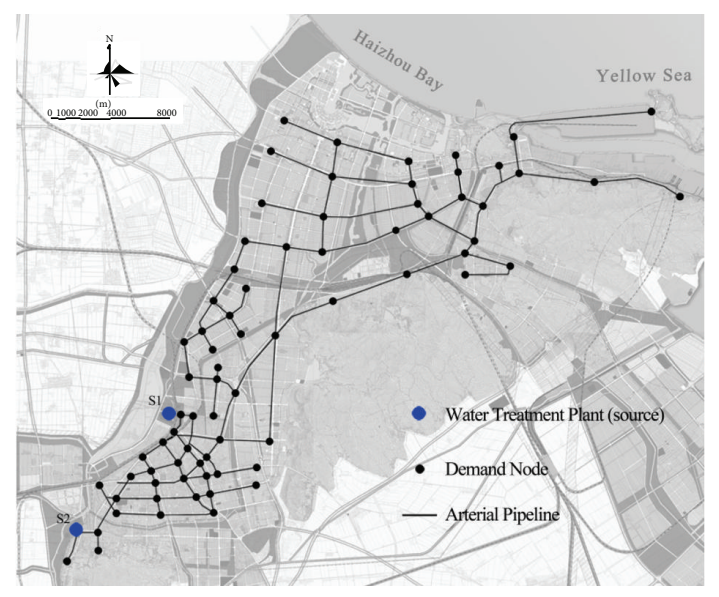

FIGURE 4: The graphical representation of original WDN in Lianyungang City.

pipelines are made of ductile materials (PVC) now. Due to the pipe size limit and geological condition, it is unlikely to replace all pipelines with ductile materials. According to the current municipal planning, ductile pipelines can reach almost 75\% after taking ductile retrofitting strategy.

As a stage of WDN expansion, meshed expansion is a proper way to achieve the system optimization purpose. As shown in Figure 5, the evolution and expansion of a WDN can be roughly divided into three stages [7]: branched expansion, looped expansion, and meshed expansion.

Branched Expansion. The notion is to develop a WDN configuration where one directional flow can only be transmitted from the source to demand nodes, with each intermediate node connected to one upstream and one downstream node only.

Looped Expansion. The notion is to develop a WDN configuration consisting of the nodes that can receive water from more than one source as a consequence of a loop structure. It is created by connecting the end nodes of the branched network to set up loops.

Meshed Expansion. The notion is to develop an almost totally looped expansion WDN, with most of the intermediate nodes connected to four pipes to form a grid-like structure.

Among the three stages of WDN evolution, branched expansion is the primary stage, while looped expansion and meshed expansion are the advanced stages of WDN development. Nowadays, the WDN of a common city in developing country has more or less completed the looped expansion stage, such as the municipal WDN in Lianyungang City, China (Figure 4). Therefore, as a network optimization strategy, meshed expansion is typical, adaptive, and easy to be implemented. The graphical representation of WDN under meshed expansion in Lianyungang City is shown in Figure 6.

4.3. Regional Seismic Intensity and Scenario Earthquake. Based on the study of Bruneau et al. in 2003 [19], 


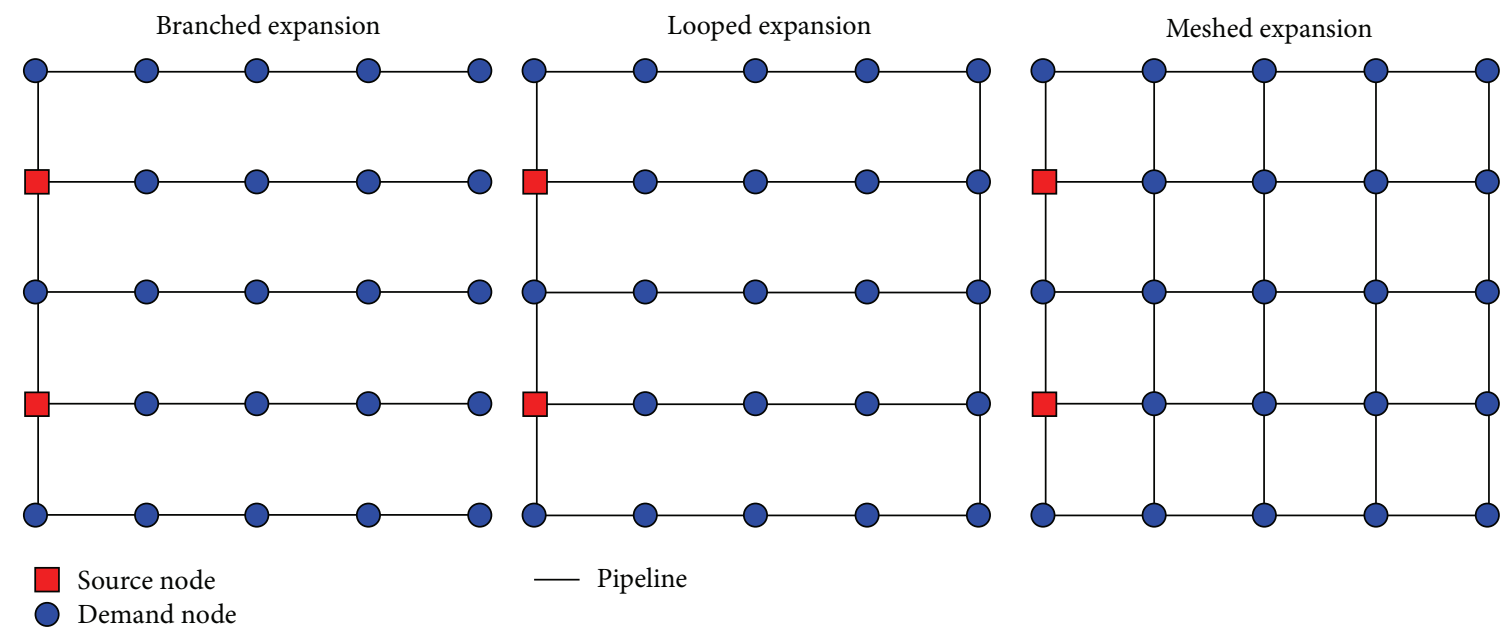

FIGURE 5: Topology illustration of three expansion stages of WDN.

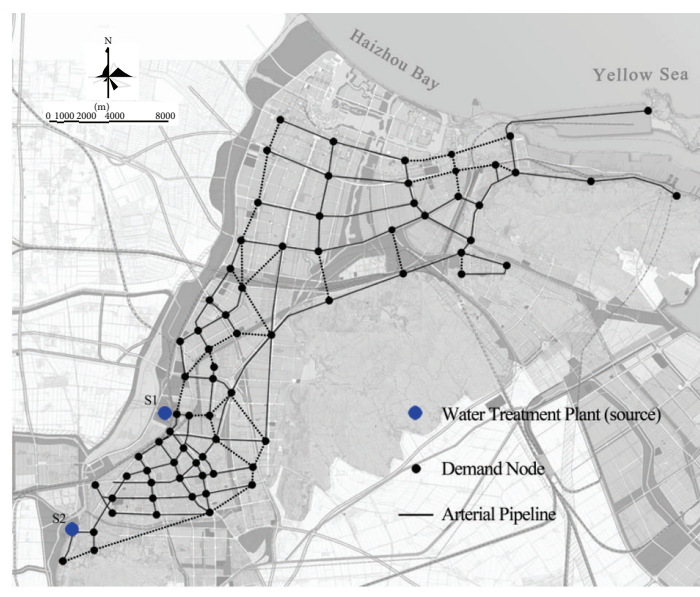

FIGURE 6: The graphical representation of WDN under meshed expansion in Lianyungang City.

the occurrence probability of disaster is a feature that should be taken into consideration in resilience assessment. For seismic disaster, Probabilistic Seismic Hazard Analysis (PSHA) is a commonly used tool that can be applied to seismic risk or reliability assessment. This tool can estimate mean annual rate of occurrence or annual probability that ground motion exceeds a specific intensity over a range of intensities [25].

However, results from PSHA are sometimes difficult for nonspecialist decision-makers to understand because they represent an aggregation of earthquake events rather than one specific earthquake [25]. What is more, the application of this tool needs detailed data and information. On the other hand, a resilience assessment based on scenario events avoids these difficulties. For the purpose of our study to compare the effects of two different kinds of reconstruction strategies on resilience, a representative scenario earthquake is enough to achieve this goal succinctly and effectively.

According to the historical data provided by the Construction Bureau of Lianyungang City, the rare intensity of this city is VIII degree. In other words, over a design
TABLE 2: The basic ground motion parameters of scenario earthquake.

\begin{tabular}{lc}
\hline Seismic intensity & VIII \\
\hline Corresponding ground motion parameters & \\
Peak Ground Acceleration $(\mathrm{g})^{\mathrm{a}}$ & 0.3602 \\
Peak Ground Velocity $(\mathrm{m} / \mathrm{s})$ & 0.35 \\
\hline${ }^{\mathrm{a}}$ The unit $\mathrm{g}$ is gravitational acceleration $\left(\mathrm{g} \approx 9.81 \mathrm{~m} / \mathrm{s}^{2}\right)$. &
\end{tabular}

reference period of 50 years, the probability of earthquakes exceeding VIII is $2 \%$. The rare seismic intensity is a basis for seismic-resistance design of buildings and infrastructures under disadvantage condition. Therefore, it is appropriate to choose the VIII degree seismic intensity as scenario event. The Peak Ground Acceleration (PGA) and Peak Ground Velocity $(\mathrm{PGV})$ at this scenario earthquake are listed in Table 2.

4.4. Seismic Fragility Analysis of Pipeline Components. The municipal WDN in Lianyungang City is made up of point components and pipeline components. It can be assumed that the point components, namely, the two water treatment plants $S_{1}$ and $S_{2}$, can maintain basic functions in seismic disasters provided that the main devices and backup power are undamaged and function well [5]. Therefore, the seismic fragility analysis mainly refers to the pipeline components.

For the pipelines in arterial WDN, the extent of damage was measured by the number of broken points per kilometer, which is subject to the Permanent Ground Failure (PGD) and Peak Ground Velocity (PGV) at scenario seismic intensity VII [27]. The numbers of broken points per kilometer of each pipeline should be calculated for both factors separately and the largest value taken to represent the extent of the damage.

In detail, according to the HAZUS-MR4 [27], the seismic fragility model associated with PGV is provided as follows:

Break Rate [Breaks/km]

$$
\cong \alpha \times 3.1623 \times 10^{-9} \times(\mathrm{PGV})^{(2.25)} \text {, }
$$




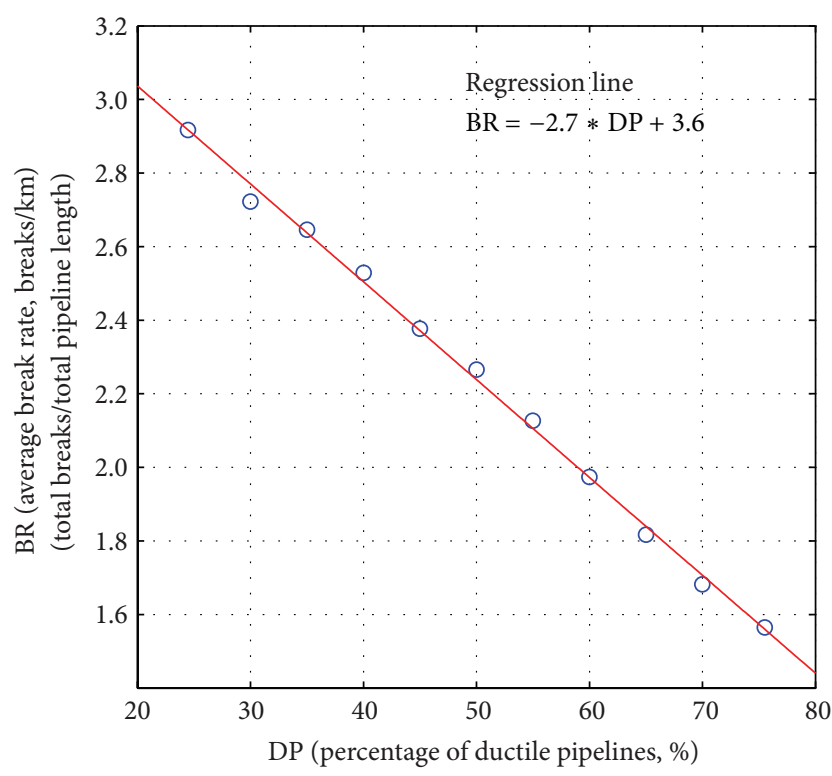

FIGURE 7: The variation of average break rate with percentage of ductile pipelines.

where $\alpha$ refers to the material parameter. For pipelines made of brittle materials, $\alpha=1$, while for pipeline made of ductile materials, $\alpha=0.3$. With PGV expressed in $\mathrm{m} / \mathrm{sec}$, this model is based on the empirical data corresponding to actual pipeline damage observed in four US and two Mexican earthquakes [32]. The break rate of each pipeline due to PGV can be determined according to (3).

Then the seismic fragility model associated with PGD is based on work conducted by Honegger and Eguchi (1992) [33] for the San Diego County Water Authority (SDCWA). This fragility model is provided as follows:

$$
\begin{aligned}
& \text { Break Rate }[\text { Breaks } / \mathrm{km}] \\
& \cong \alpha \times \operatorname{Prob}[\mathrm{Liq}] \times 0.1279 \times(\mathrm{PGD})^{(0.56)},
\end{aligned}
$$

where $\alpha$ refers to the material parameter as defined in (3). PGD is expressed in meter, which is determined by site susceptibility category, Peak Ground Acceleration (PGA) [27]. Prob[Liq] is the probability of liquefaction, which is influenced by Peak Ground Acceleration (PGA), groundwater depth, and site susceptibility category [27]. The main urban area of Lianyungang is quaternary covering Holocene sea plane, which is moderately susceptible to liquefaction. Based on the PGA provided in our scenario earthquake and groundwater depth distribution data of Lianyungang City, the break rate of each pipeline due to PGD can be determined according to (4).

According to (3) and (4), the break rate of each pipeline can be exactly calculated. Because ductile material will decrease the break rate of each pipeline, the damage extent of entire WDN is subject to the percentage of ductile pipelines. Figure 7 shows the variation of average break rate (total breaks/total pipeline length) with the percentage of ductile pipelines.
(1)

(4)

(6)

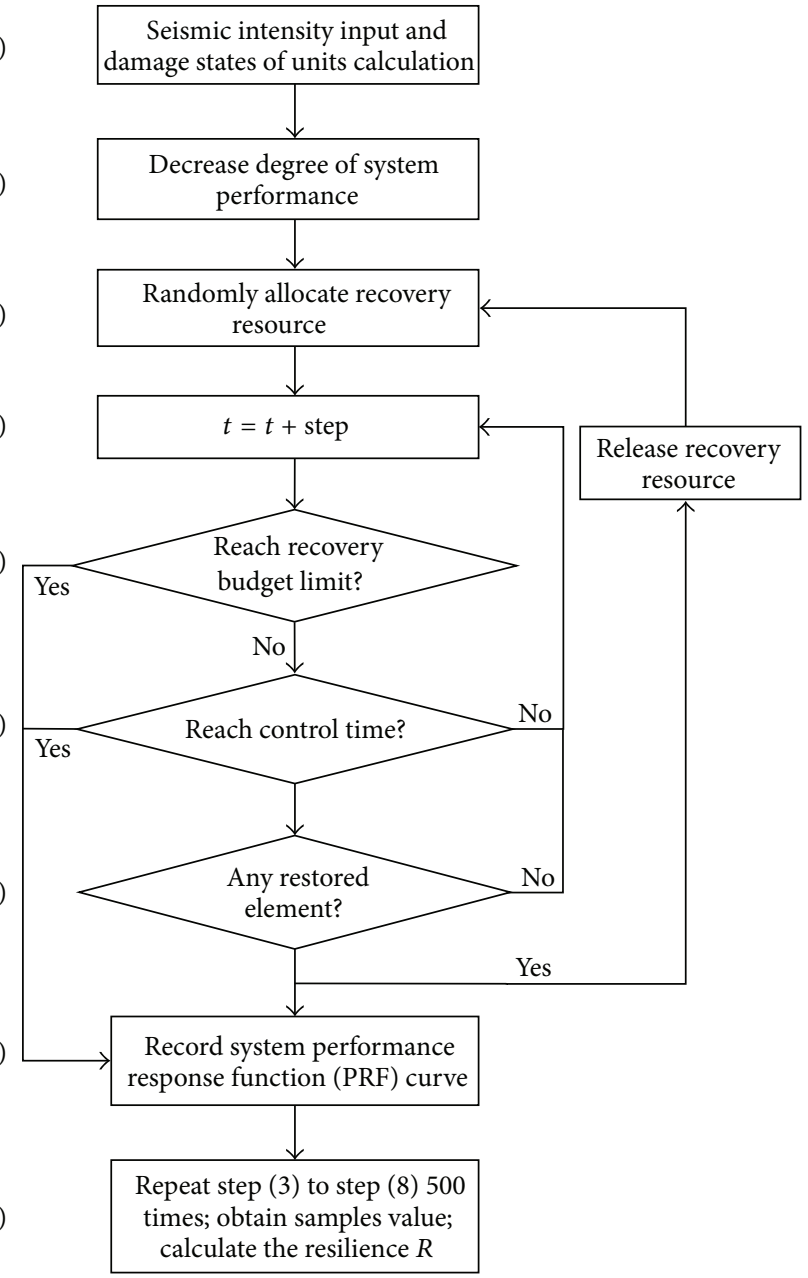

FIGURE 8: Simulation procedure for executing resilience assessment.

From Figure 7, it can be seen that the average break rate of WDN linearly deceases with percentage of ductile pipelines. The average break rate of WDN will reduce to a minimum when the ductile retrofitting strategy is strictly executed (100\% ductile pipelines). Therefore, given the same recovery conditions, the resilience value of WDN under complete ductile retrofitting strategy will be higher than that with 75 percent of ductile pipelines. But, in this case, ductile pipelines can only reach almost $75 \%$ after taking ductile retrofitting strategy due to practical constraints. Thus, it is necessary to announce that the resilience enhancing effect of ductile retrofitting strategy in this case is somewhat conservative.

4.5. Simulation Procedure to Execute the Resilience Assessment. Figure 8 depicts the simulation procedure for assessing resilience of WDNs. The key point of this procedure is to generate the time-dependent PRF curve with the constraints of recovery conditions.

To help understand and implement the procedure, the key aspects of the simulation are discussed as follows:

(1) For the PRF $\varepsilon_{5}(t)$ which integrates both technical dimension and organizational dimension in Table 1, 
$C_{\omega}(t)$ is a key parameter for calculating PRF. In the ideal situation, $C_{\omega}(t)$ should be defined as the frictional head loss between the source node and demand node in $S / D$ pair $\omega$. However, the observed water head data are usually insufficient in practice. Therefore, $C_{\omega}(t)$ is simplified into the shortest path length between $S$ and $D$ in this study because the path length is directly proportional to the frictional head loss. In addition, the network equilibrium theory suggested that it is reasonable to take the shortest path length as the generic minimal path $\operatorname{cost} C_{\omega}(t)$ [30].

(2) The recovery resources were nondimensionalized as the number of "resource units" due to the lack of adequate information on local recovery efficiency. A resource unit includes a complete 16-person crew and associated repair equipment. Therefore, it can be estimated that a pipeline break can be restored by a resource unit in 3 hours [27]. Similarly, the local recovery budgets were nondimensionalized as the number of "budget units." A budget unit represents the recovery budgets needed for repairing a pipeline break.

(3) In order to reflect the state of disorder during the recovery process right after seismic disaster, stochastic strategy was used to allocate recovery resources. By using this strategy, the restoration sequence was generated randomly, and then the available resource units were allocated according to the generated restoration sequence. For each restoration sequence, by going through steps (3) to (8), a network PRF curve can be recorded, and a value of resilience can be calculated. To obtain enough sample values, 500 restoration sequences were generated randomly, and 500 system PRF curves and 500 values of resilience were obtained by iterating the calculation through steps (3) to (8) for 500 times. The final value of resilience $R$ was the mean of the 500 sample values.

(4) According to the fresh water reserves of residents in Lianyungang, the control time $T_{R}$ was set to 5 days. The working time of recovery was set to 12 hours per day, and then the control time $T_{R}$ equals 60 hours.

\section{Comparison Results}

In different dimensions (technical dimension, organizational dimension, and the combination of these two dimensions), we analyzed and contrasted the effects of two types of reconstruction strategies, namely, meshed expansion and ductile retrofitting, on enhancing the seismic resilience of the municipal WDN in Lianyungang City.

\subsection{Resilience Enhancing Effects in Technical Dimension. In} this section, the effects of two reconstruction strategies on enhancing the seismic resilience of the municipal WDN were contrasted in technical dimension. Technical dimension usually refers to the rate of physical unit loss [23] or the physical index of infrastructure networks $[7,22]$. In our work, we adopted the network physical index to represent technical dimension and depicted it from three typical perspectives: network structure, network redundancy, and network efficiency. As Table 1 shows, PRF $\varepsilon_{1}(t)$ refers to the network structure, $\varepsilon_{2}(t)$ refers to the network redundancy, and $\varepsilon_{3}(t)$ provides a limited view of network efficiency.

From the perspective of network structure ( $\left.\operatorname{PRF} \varepsilon_{1}(t)\right)$, it can be observed that the ductile retrofitting is an effective strategy to significantly improve the resilience of WDN, especially when the recovery budget $b$ is not sufficient. At given 300 budget units and 500 budget units, the resilience variation curve of WDN under ductile retrofitting with recovery resource $r$ is obviously higher than that of original WDN state and WDN under meshed expansion (Figure 9(a)). When the recovery budget input is sufficient ( $b=1000$ budget units), resilience variation curves of three WDN states with recovery resource $r$ are very close to each other (Figure 9(a)). At any given resource $r$, the resilience variation curve of WDN under ductile retrofitting with recovery budget $b$ is significantly higher than that of the other two WDN states (Figure 9(b)). When the recovery budget increases to 1000 units, the values of resilience $R$ of three WDN states become very close to each other (Figure 9(b)).

Comparing with ductile retrofitting, the effect of meshed expansion on resilience is not obvious from the perspective of network structure (PRF $\left.\varepsilon_{1}(t)\right)$. At any combination of recovery resource $r$ and recovery budget $b$, the value of resilience $R$ of WDN under meshed expansion is pretty close to that of original WDN state (Figures 9(a) and 9(b)).

When it comes to the perspective of network redundancy (PRF $\varepsilon_{2}(t)$ ), the resilience variation of three different WDN states with $b$ and $r$ is very similar to that from the perspective of network structure (Figures 9 and 10). It means that, from the perspective of network redundancy, ductile retrofitting is also an effective strategy to obviously improve the resilience of WDN when the recovery budget $b$ input is not sufficient, while the effect of meshed expansion still did not show apparent effect on resilience.

Figure 11 provides the resilience variation of different WDN states with resource $r$ (Figure 11(a)) and budget $b$ (Figure 11(b)) from the perspective of network efficiency $\left(\operatorname{PRF} \varepsilon_{3}(t)\right)$. It can be seen that ductile retrofitting is still a highly effective strategy to obviously improve the resilience of WDN from the perspective of network efficiency. Different from the perspective of network structure and network redundancy, meshed expansion will partly enhance the resilience of WDN from the perspective of network efficiency when the recovery resource $r$ and recovery budget $b$ are relatively abundant. As shown in Figure 11, when the recovery resource $r$ and recovery budget $b$ input is scarce $(r<$ 50 resource units, $b<300$ budget units), the difference between the resilience values $R$ of WDN under meshed expansion and original WDN state is quite modest. Then with the recovery resource $r$ and recovery budget $b$ increasing, the difference becomes more pronounced. Finally when the recovery resource $r$ and recovery budget $b$ input is sufficient ( $r>100$ resource units, $b>800$ budget units), the resilience value $R$ of WDN under meshed expansion becomes nearly equal to that of original WDN state. 

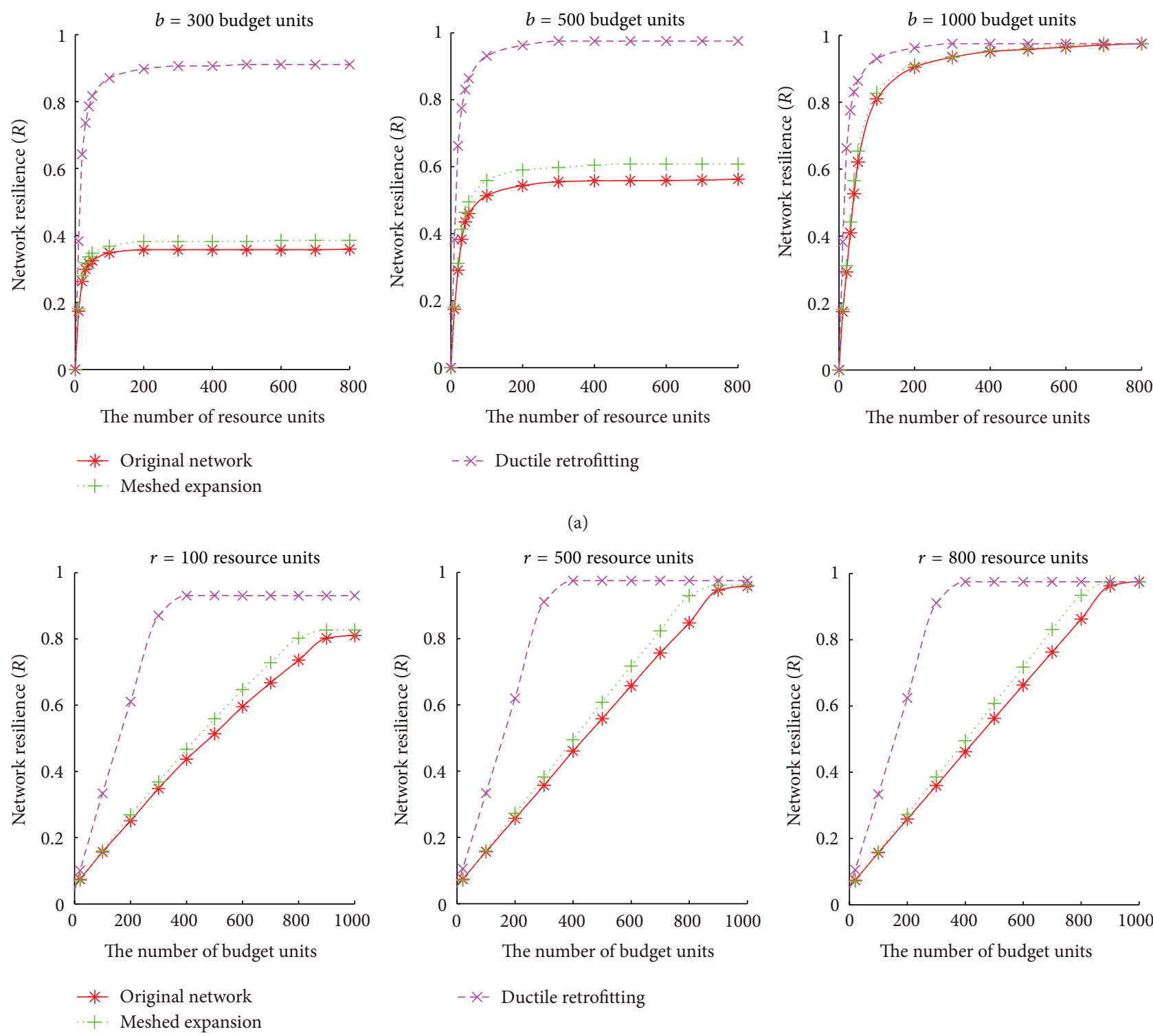

(b)

FIGURE 9: $\operatorname{PRF} \varepsilon_{1}(t)$ : variation of resilience $R$ under different reconstruction strategies with (a) recovery resource $r$ by given recovery budget $b$ and (b) recovery budget $b$ by given different recovery resource $r$.

5.2. Resilience Enhancing Effects in Organizational Dimension. The effects comparison of two reconstruction strategies on organizational resilience is shown in Figure 12. The resilience in organizational dimension was measured from the perspective of water supply rate as PRF $\varepsilon_{4}(t)$ shows in Table 1 .

From the perspective of water supply rate, the resilience variation of three different WDN states with $b$ and $r$ is very similar to that from the perspective of network efficiency (Figures 12 and 11). Therefore, it can be observed that ductile retrofitting can significantly improve the organizational resilience of $\mathrm{WDN}$, while meshed expansion will partly enhance the resilience of WDN from the perspective of water supply rate when the recovery resource $r$ and budget $b$ are relatively sufficient.
The organizational performance of WDN in this study refers to the service function, namely, the water supply rate, which largely depends on physical network status that belongs to technical dimension [23]. The variation tendency and resilience value in Figure 12 is very close to that in Figure 11. Thus, compared with link density and meshedness coefficient, the organizational resilience of WDN is much more pertinent to the average transmission-length. However, organizational performance also depends on network flow conditions, and the interaction between technical and organizational dimension is complex [23]. The focus of this study is to propose a framework to contrast the resilience enhancing effects of two reconstruction strategies in different dimensions, so the interaction mechanism between technical 

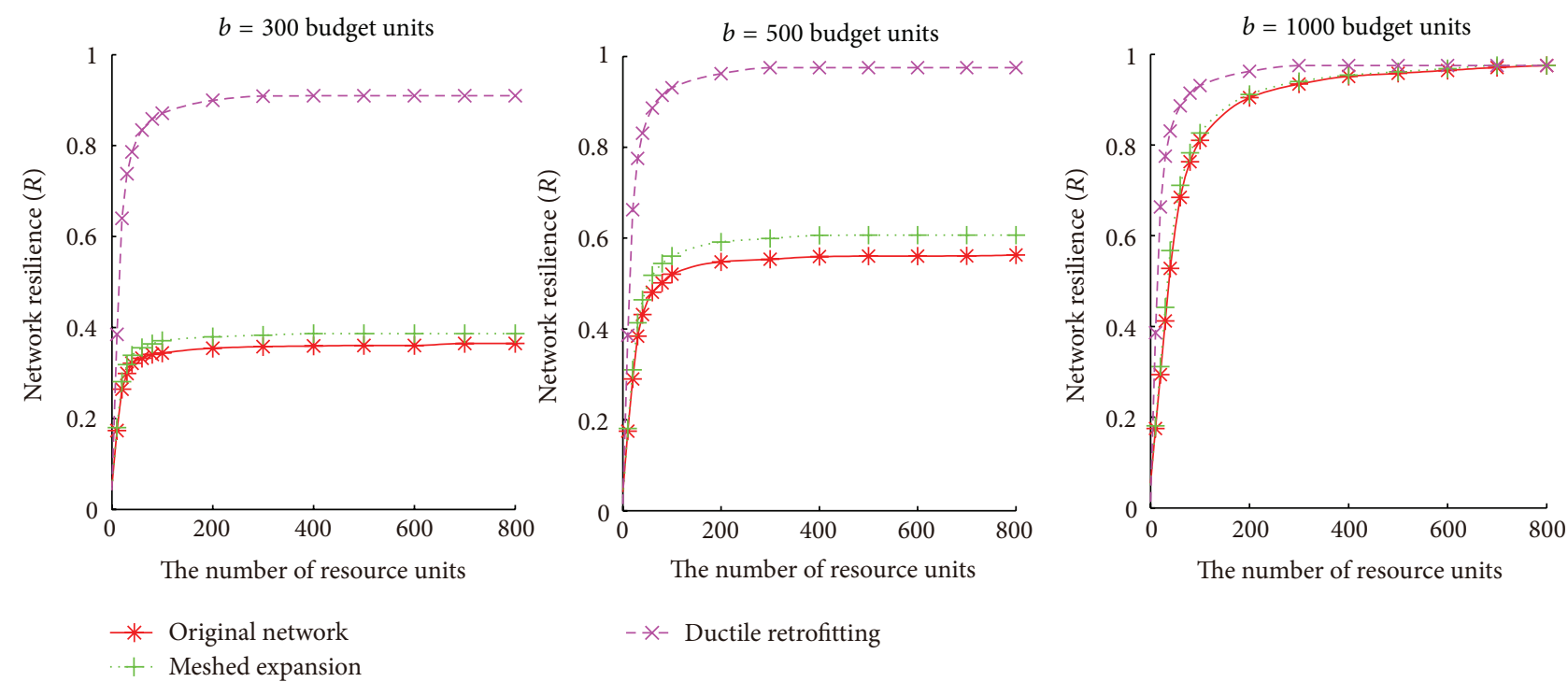

(a)
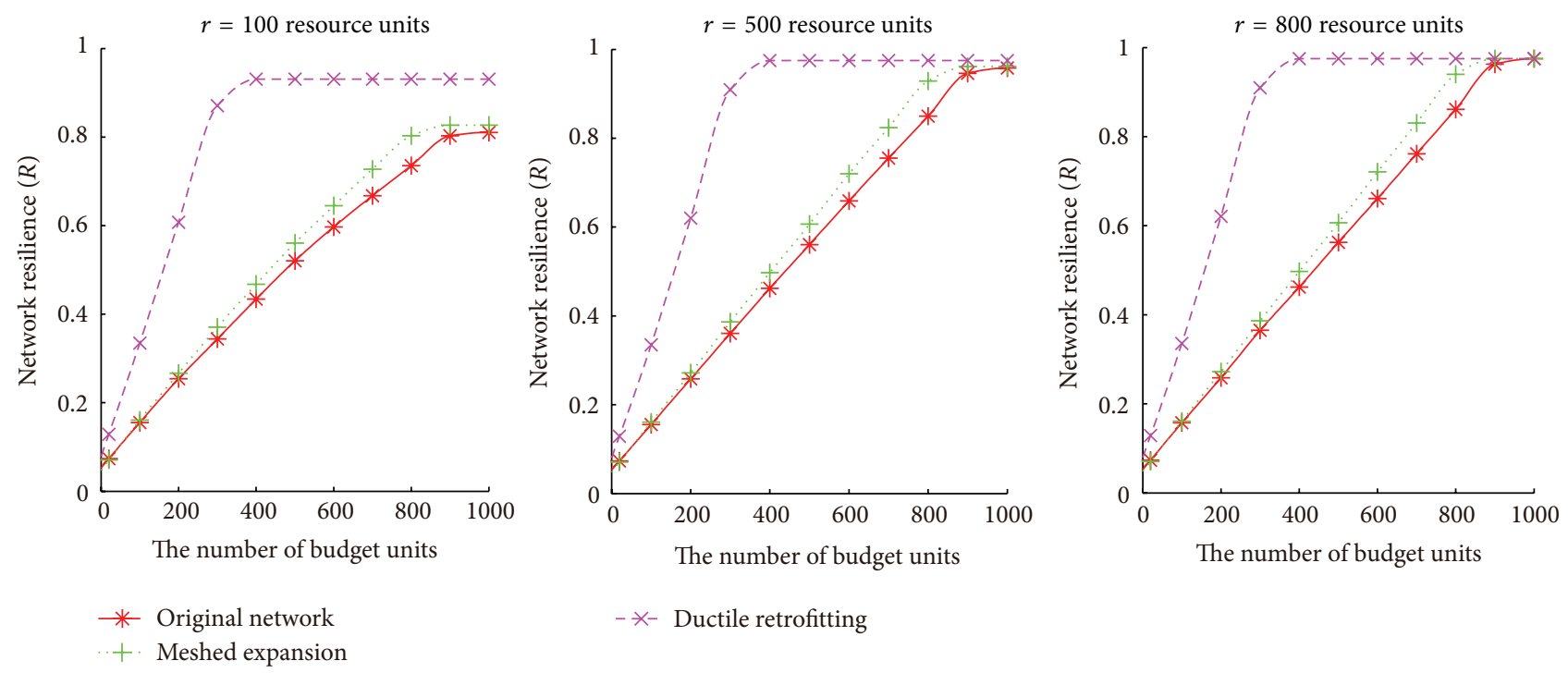

(b)

FIGURE 10: $\operatorname{PRF} \varepsilon_{2}(t)$ : variation of resilience $R$ under different reconstruction strategies with (a) recovery resource $r$ by given recovery budget $b$ and (b) recovery budget $b$ by given different recovery resource $r$.

and organizational resilience needs more theoretical research in the future.

\subsection{Resilience Enhancing Effects in Integrated Technical and} Organizational Dimension. Based on network equilibrium theory, we developed PRF $\varepsilon_{5}(t)$ to assess the resilience of three different WDN states under different reconstruction strategies. In this PRF, the technical dimension is described by the generic minimal path cost which has to follow Wardrop's first equilibrium principle [31] to reflect the change of physical topology status during the recovery process, while the organizational dimension is represented by the service demand that can be supplied in each $S / D$ pair.

The effects comparison of two reconstruction strategies on seismic resilience in integrated dimension is shown in
Figure 13. From the perspective of integration of the two dimensions, ductile retrofitting remains an effective strategy to significantly improve the resilience of WDN. The resilience variation curve of WDN under ductile retrofitting with recovery resource $r$ (Figure 13(a)) and recovery budget $b$ (Figure 13(b)) is obviously higher than that of original WDN state and WDN under meshed expansion.

However, different from ductile retrofitting, the meshed expansion strategy makes no contribution to enhancement of the seismic resilience in integrated technical and organizational dimension. Instead, this kind of strategy will diminish it to some extent. As shown in Figure 13, when the recovery resource $r$ and recovery budget $b$ input is scarce $(r<50$ resource units, $b<300$ budget units), the resilience $R$ of WDN under meshed expansion is very close to that of 

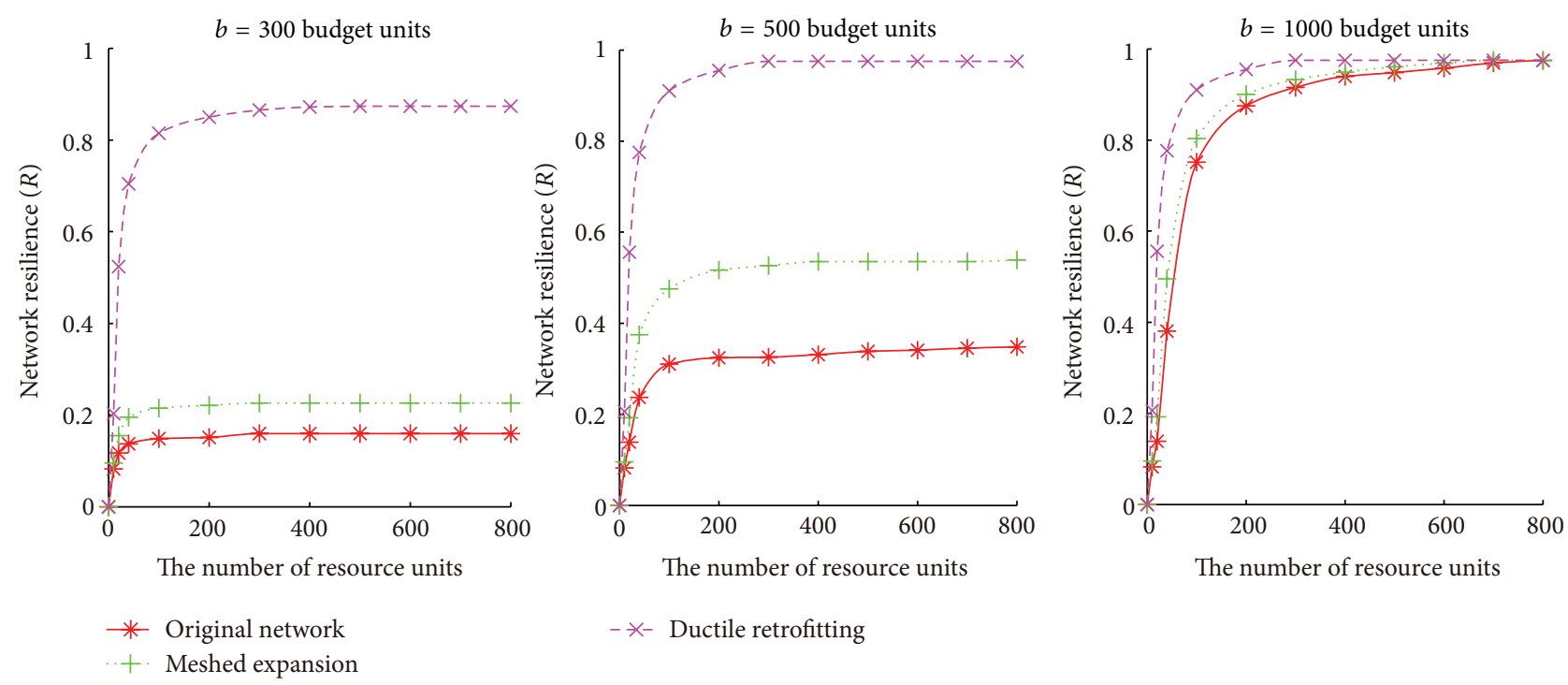

(a)
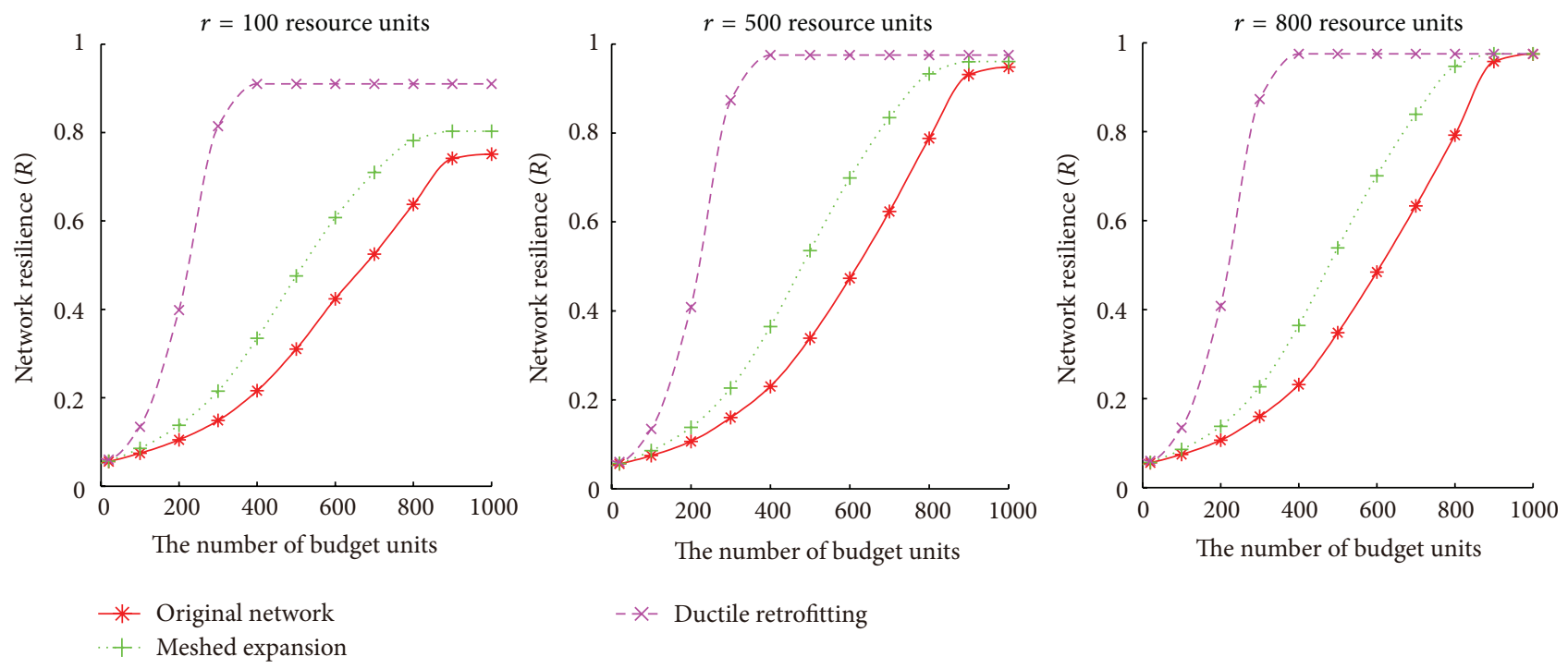

(b)

FIGURE 11: PRF $\varepsilon_{3}(t)$ : variation of resilience $R$ under different reconstruction strategies with (a) recovery resource $r$ by given recovery budget $b$ and (b) recovery budget $b$ by given different recovery resource $r$.

original WDN state. Then, with the recovery resource $r$ and recovery budget $b$ increasing, the resilience $R$ of WDN under meshed expansion begins to be obviously lower than that of original WDN state. Finally, when the recovery resource $r$ and recovery budget $b$ input is abundant $(r>100$ resource units, $b>800$ budget units), the resilience value $R$ of WDN under meshed expansion becomes nearly equal to that of original WDN state again.

5.4. Analysis and Discussion. In the foregoing, we explored and contrasted the effects of two different reconstruction strategies-ductile retrofitting strategy and meshed expansion strategy - on the seismic resilience of municipal WDN. The results have shown that the ductile retrofitting strategy is an effective strategy to significantly improve the resilience of
WDN in technical dimension and organizational dimension. Even in the integrated dimension of these two dimensions, the ductile retrofitting strategy is also an efficient way to enhance the seismic resilience of municipal WDN.

The main reason why the ductile retrofitting strategy is very helpful on seismic resilience is that it will enhance earthquake resistance of pipelines. Theoretically, ductile retrofitting will reduce the breakage ratio of pipelines by up to 70 percent in seismic disasters [27]. Therefore, this strategy is able to improve the postquake performance of WDN $\left(P_{1}\right.$ in Figure 1) with more still-working pipe units right after the seismic disaster. What is more, the low breakage ratio of pipelines will significantly reduce the recovery burden, namely, the recovery budget and recovery resource needed in the restoration process. For these two advantages, the WDN 

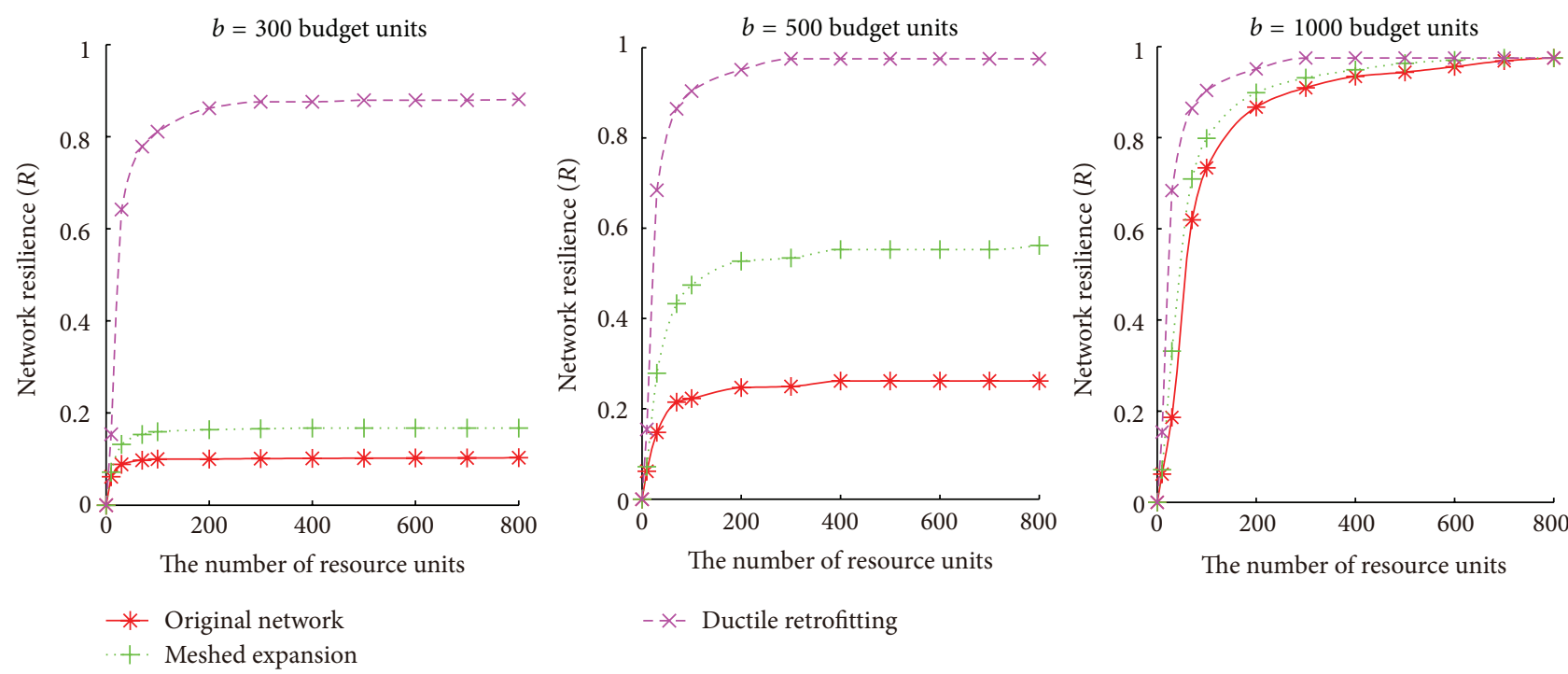

(a)
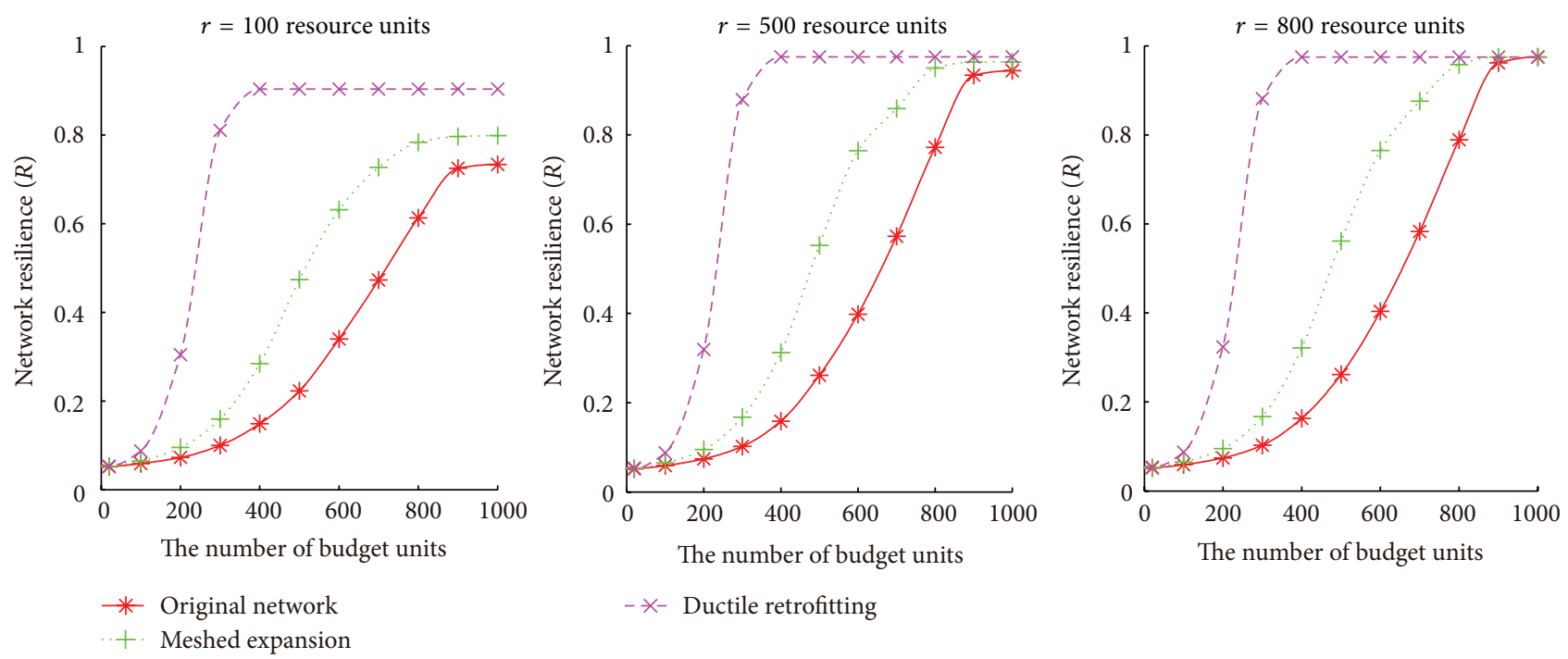

(b)

FIGURE 12: $\mathrm{PRF} \varepsilon_{4}(t)$ : variation of resilience $R$ under different reconstruction strategies with (a) recovery resource $r$ by given recovery budget $b$ and (b) recovery budget $b$ by given different recovery resource $r$.

under ductile retrofitting can get higher seismic resilience than the other two WDN states, especially when the recovery budget input is insufficient.

Comparing with ductile retrofitting strategy, the contribution of meshed expansion strategy to seismic resilience is nonsignificant in technical and organizational dimension. Even this kind of strategy can lead to a decline in the seismic resilience of WDN, with the constraints of recovery budget and recovery resource. This phenomenon can be interpreted from two aspects. First, by taking the meshed expansion strategy, both PRF $\varepsilon(t)$ and the pre-event normal performance $p(t)$ can be improved. As a result, the seismic resilience can possibly be reduced, referring to (1) for details. Second, by taking the meshed expansion strategy, the additional pipelines will increase the burden of recovery with the constraints of recovery budget and recovery resource.
Although the meshed expansion strategy will reduce the seismic resilience of municipal WDN, it is worth noting that this strategy can contribute to the construction of a new network topology with higher performance level from preevent time to the end of restoration process, while the ductile retrofitting strategy has no advantage on this. Therefore, the meshed expansion strategy is also an alternative when the standby recovery budget and resource are sufficient and abundant.

5.5. Research Limitation and Future Study. Generally speaking, the units retrofitting strategy has many reconstruction measures such as ductile retrofitting of pipelines [5], structure reinforcement of water treatment plant [6], and power backup of pumping station [5], while the network optimization strategy includes network meshed expansion [7], 

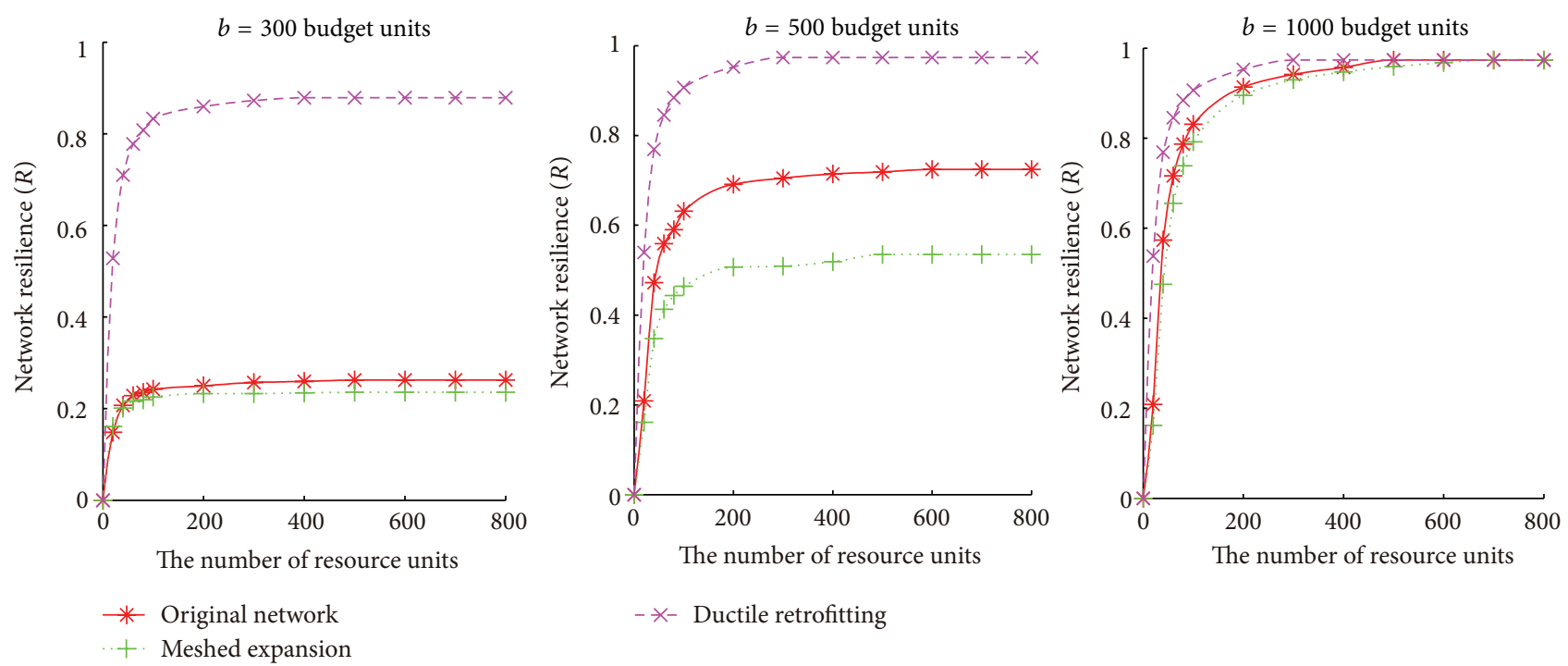

+ Meshed expansion

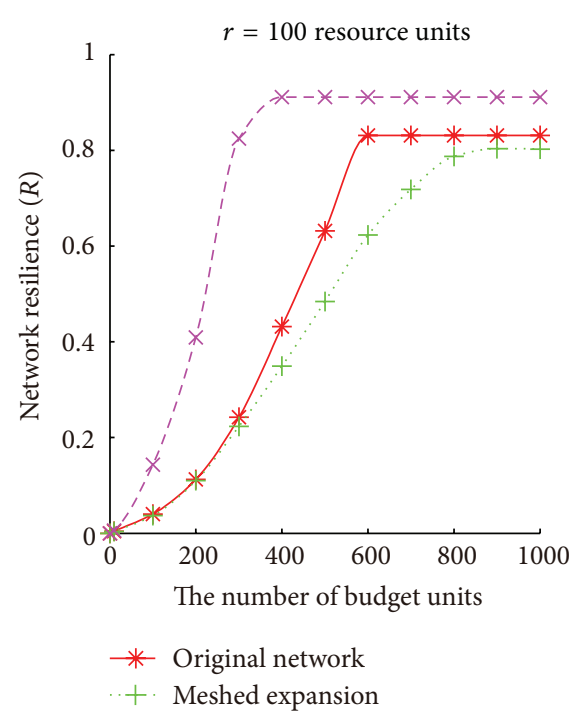

(a)
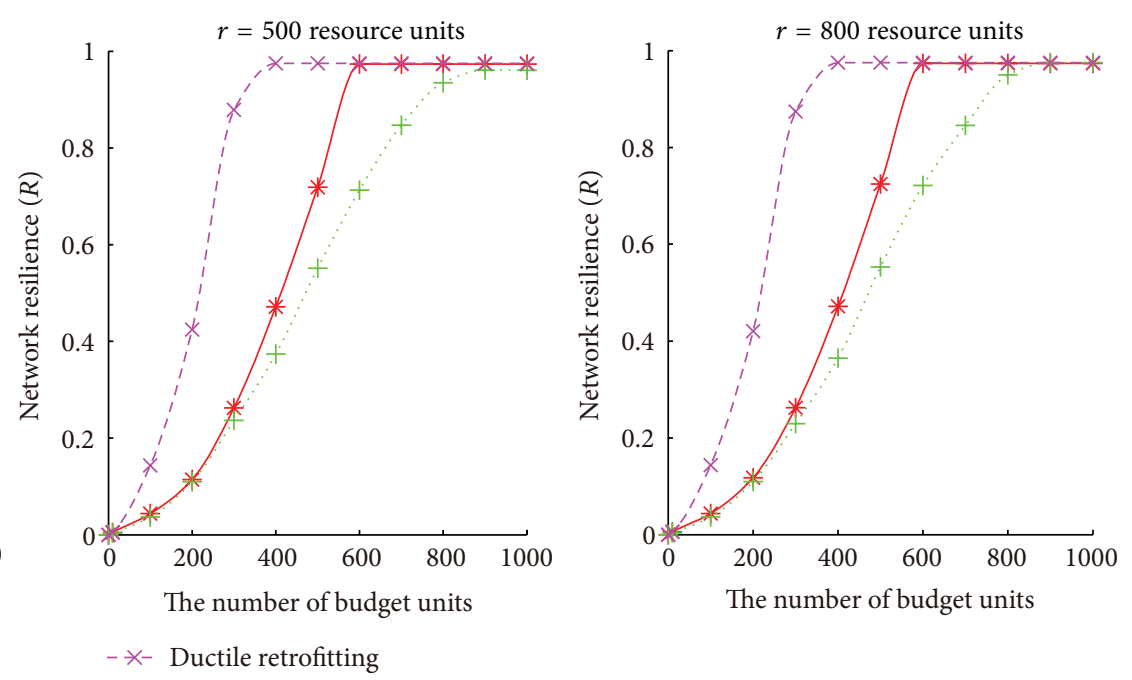

(b)

FIGURE 13: $\mathrm{PRF} \varepsilon_{5}(t)$ : variation of resilience $R$ under different reconstruction strategies with (a) recovery resource $r$ by given recovery budget $b$ and (b) recovery budget $b$ by given different recovery resource $r$.

redundancy design [8], and self-healing and self-adapting system response mechanisms [9]. In this work, we chose two typical and widely applied reconstruction measures and emphasized proposing a feasible comparison framework to demonstrate the enhancing effects of these two strategies on WDN resilience. In the future, we will look to explore the other measures and offer a thorough comparison of these two kinds of strategies.

\section{Conclusions}

For the municipal WDN, we proposed a feasible framework to contrast the effects of two different reconstruction strategies on improving the seismic resilience. The effects of ductile retrofitting strategy and meshed expansion strategy on seismic resilience of WDN were first contrasted in two dimensions (technical and organizational). What is more, taking the interrelationship between the two dimensions into account, we also developed a measurement integrating the two dimensions to contrast the resilience enhancing effects of these two strategies.

Through the case study of municipal WDN in Lianyungang City, China, resilience variation curves of three different WDN states (original WDN state, WDN under ductile retrofitting, and WDN under meshed expansion) with recovery budget $b$ and recovery resource $r$ are plotted and analyzed. From the results of this case study, we drew the following conclusions.

The ductile retrofitting strategy is an effective strategy to significantly improve the resilience of WDN in multidimensions, including the technical dimension, the organizational 
dimension, and the integrated dimension of these two dimensions.

The effect of meshed expansion strategy on seismic resilience of WDN is nonsignificant in technical and organizational dimension. For the integrated dimension of these two dimensions, it will even lead to a decline in the seismic resilience of WDN.

Although the meshed expansion strategy has no obvious effect on improving the seismic resilience of WDN, this strategy can contribute to the construction of a new network topology with higher performance level from pre-event time to the end of restoration process, while the ductile retrofitting strategy has no advantage on this.

For decision-makers, the ductile retrofitting is a preferred strategy to enhance the seismic resilience of WDN when the recovery budget and recovery resource are insufficient. While the standby recovery budget and resource are abundant, the meshed expansion is also an alternative strategy which can promote the performance of WDN network.

\section{Conflict of Interests}

The authors declare that there is no conflict of interests regarding the publication of this paper.

\section{Acknowledgments}

The authors appreciate the data and information supports from the City Construction Bureau, Water Supply General Company, and Municipal Design and Research Institute of Lianyungang City, China.

\section{References}

[1] J. Moteff, C. Copeland, and J. Fischer, "Critical infrastructures: what makes an infrastructure critical?" Congress Report, The Library of Congress, Washington, DC, USA, 2003.

[2] UNDP, "Scarcity: power, poverty and global water crisis," Human Development Report, UNDP, New York, NY, USA, 2006.

[3] W. Liu, Y. G. Zhao, and J. Li, "Seismic functional reliability analysis of water distribution networks," Structure and Infrastructure Engineering, vol. 11, no. 3, pp. 363-375, 2015.

[4] M. Kobayashi, "Experience of infrastructure damage caused by the Great East Japan Earthquake and countermeasures against future disasters," IEEE Communications Magazine, vol. 52, no. 3, pp. 23-29, 2014.

[5] S. Esposito, S. Giovinazzi, L. Elefante, and I. Iervolino, "Performance of the L'Aquila (central Italy) gas distribution network in the $2009\left(\mathrm{M}_{\mathrm{w}}\right.$ 6.3) earthquake," Bulletin of Earthquake Engineering, vol. 11, no. 6, pp. 2447-2466, 2013.

[6] G\&E, NIBS Earthquake Loss Estimation Methods, Technical Manual (Water Systems), Engineering Systems Inc, 1994.

[7] A. Yazdani, R. A. Otoo, and P. Jeffrey, "Resilience enhancing expansion strategies for water distribution systems: a network theory approach," Environmental Modelling and Software, vol. 26, no. 12, pp. 1574-1582, 2011.

[8] M. Ouyang, M.-H. Yu, X.-Z. Huang, and E.-J. Luan, "Emergency response to disaster-struck scale-free network with redundant systems," Physica A: Statistical Mechanics and its Applications, vol. 387, no. 18, pp. 4683-4691, 2008.

[9] S. Pahwa, A. Hodges, C. Scoglio, and S. Wood, “Topological analysis of the power grid and mitigation strategies against cascading failures," in Proceedings of the 4th Annual IEEE International Systems Conference (SysCon '10), pp. 272-276, April 2010.

[10] K. Pitilakis, M. Alexoudi, S. Argyroudis, O. Monge, and C. Martin, "Earthquake risk assessment of lifelines," Bulletin of Earthquake Engineering, vol. 4, no. 4, pp. 365-390, 2006.

[11] T. Imai, S. Wada, and T. Koike, "Seismic risk assessment and mitigation for the existing lifeline," Journal of Earthquake and Tsunami, vol. 5, no. 1, pp. 31-45, 2011.

[12] K. Poljanšek, F. Bono, and E. Gutiérrez, "Seismic risk assessment of interdependent critical infrastructure systems: the case of European gas and electricity networks," Earthquake Engineering and Structural Dynamics, vol. 41, no. 1, pp. 61-79, 2012.

[13] A. S. Selçuk and M. S. Yücemen, "Reliability of lifeline networks under seismic hazard," Reliability Engineering and System Safety, vol. 65 , no. 3, pp. 213-227, 1999.

[14] A. S. Selçuk and M. Semih Yücemen, "Reliability of lifeline networks with multiple sources under seismic hazard," Natural Hazards, vol. 21, no. 1, pp. 1-18, 2000.

[15] J. Song and S.-Y. Ok, "Multi-scale system reliability analysis of lifeline networks under earthquake hazards," Earthquake Engineering \& Structural Dynamics, vol. 39, no. 3, pp. 259-279, 2010.

[16] E. Jenelius, T. Petersen, and L.-G. Mattsson, "Importance and exposure in road network vulnerability analysis," Transportation Research Part A: Policy and Practice, vol. 40, no. 7, pp. 537560, 2006.

[17] S. Menoni, F. Pergalani, M. P. Boni, and V. Petrini, "Lifeline earthquake vulnerability assessment," in Managing Critical Infrastructure Risks: Decision Tools and Application for Port Security, NATO Science for Peace and Security Series C: Environmental Security, pp. 111-132, Springer, Dordrecht, The Netherlands, 2007.

[18] S. Menoni, F. Pergalani, M. P. Boni et al., "Lifelines earthquake vulnerability assessment: a systemic approach," in Proceedings of the 2006 NATO Advanced Research Workshop on Management Tools for Port Security, Critical Infrastructure and Sustainability, pp. 111-132, 2006.

[19] M. Bruneau, S. E. Chang, R. T. Eguchi et al., "A framework to quantitatively assess and enhance the seismic resilience of communities," Earthquake Spectra, vol. 19, no. 4, pp. 733-752, 2003.

[20] G. P. Cimellaro, A. M. Reinhorn, and M. Bruneau, "Framework for analytical quantification of disaster resilience," Engineering Structures, vol. 32, no. 11, pp. 3639-3649, 2010.

[21] M. Ouyang and L. Dueñas-Osorio, "Multi-dimensional hurricane resilience assessment of electric power systems," Structural Safety, vol. 48, pp. 15-24, 2014.

[22] M. Ouyang, L. Dueñas-Osorio, and X. Min, "A three-stage resilience analysis framework for urban infrastructure systems," Structural Safety, vol. 36-37, pp. 23-31, 2012.

[23] S. E. Chang and M. Shinozuka, "Measuring improvements in the disaster resilience of communities," Earthquake Spectra, vol. 20, no. 3, pp. 739-755, 2004.

[24] S. E. Chang, T. Mcdaniels, J. Fox, R. Dhariwal, and H. Longstaff, "Toward disaster-resilient cities: characterizing resilience of infrastructure systems with expert judgments," Risk Analysis, vol. 34, no. 3, pp. 416-434, 2014. 
[25] G. P. Cimellaro, A. De Stefano, and O. Villa, "Serviceability of natural gas distribution networks after earthquakes," Journal of Earthquake and Tsunami, vol. 7, no. 2, Article ID 1350005, 2013.

[26] L. Chen and E. M. Hooks, "Resilience: an indicator of recovery capability in intermodal freight transport," Transportation Science, vol. 46, no. 1, pp. 109-123, 2012.

[27] FEMA, Multi-Hazard Loss Estimation Methodology, Earthquake Model (HAZUS-MH MR4), Federal Emergency Management Agency (FEMA), 2003.

[28] J. Buhl, J. Gautrais, N. Reeves et al., "Topological patterns in street networks of self-organized urban settlements," European Physical Journal B, vol. 49, no. 4, pp. 513-522, 2006.

[29] V. Latora and M. Marchiori, "How the science of complex networks can help developing strategies against terrorism," Chaos, Solitons \& Fractals, vol. 20, no. 1, pp. 69-75, 2004.

[30] A. Nagurney and Q. Qiang, "A network efficiency measure with application to critical infrastructure networks," Journal of Global Optimization, vol. 40, no. 1-3, pp. 261-275, 2008.

[31] J. G. Wardrop, "Some theoretical aspects of road traffic research," in Proceedings of the Institution of Civil Engineers, Part II, pp. 352-362, 1952.

[32] M. O'Rourke and G. Ayala, "Pipeline damage due to wave propagation," Journal of Geotechnical Engineering, vol. 119, no. 9, pp. 1490-1498, 1993.

[33] D. G. Honegger and R. T. Eguchi, "Determination of relative vulnerabilities to seismic damage for San Diego County Water Authority (SDCWA) Water Transmission Pipelines," 1992. 


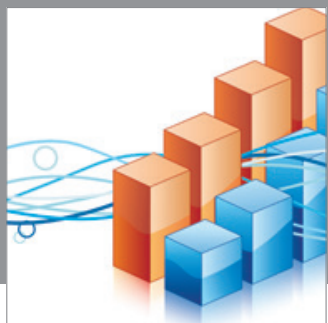

Advances in

Operations Research

mansans

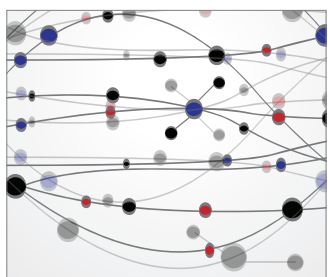

The Scientific World Journal
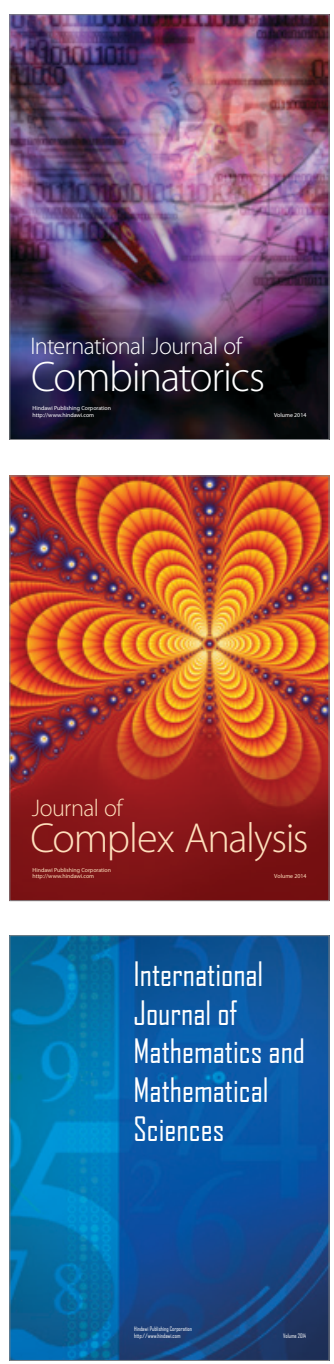
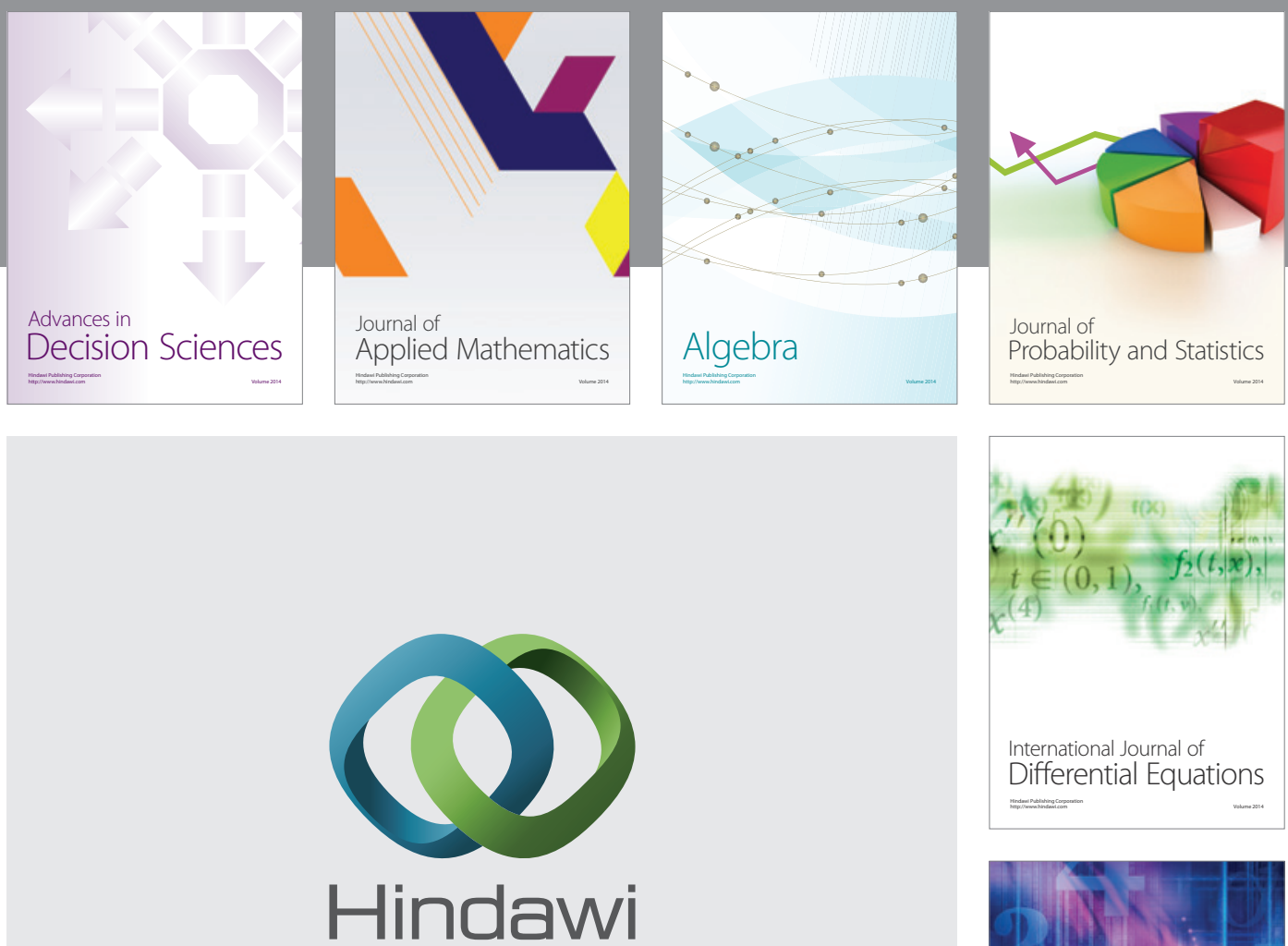

Submit your manuscripts at http://www.hindawi.com
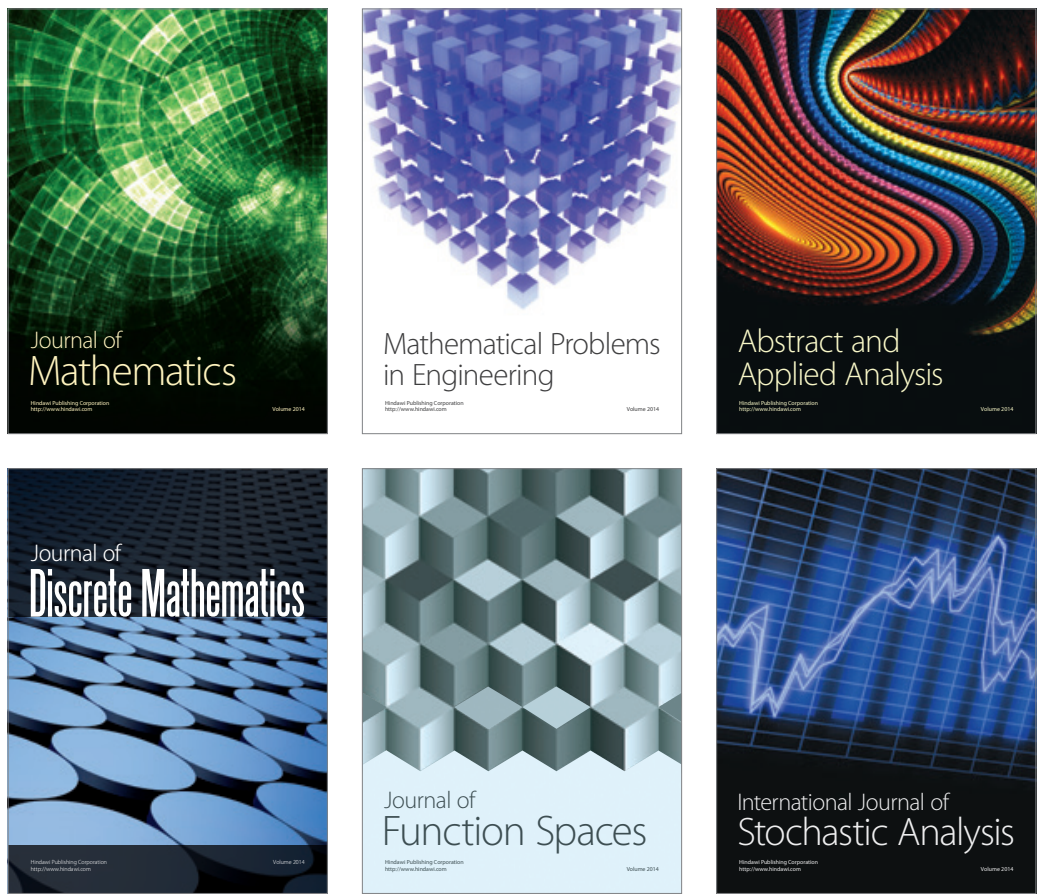

Journal of

Function Spaces

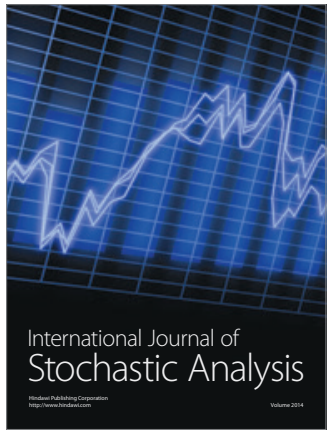

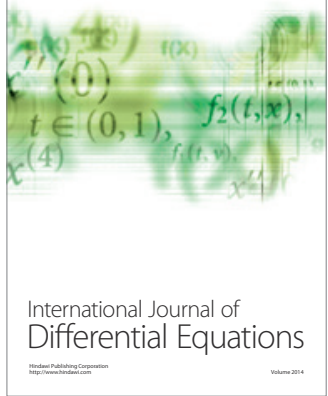
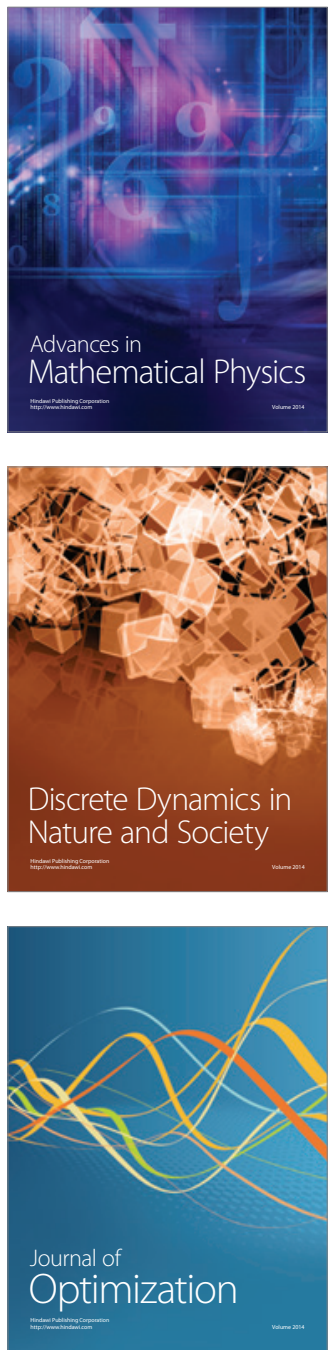